BMC

Genomics

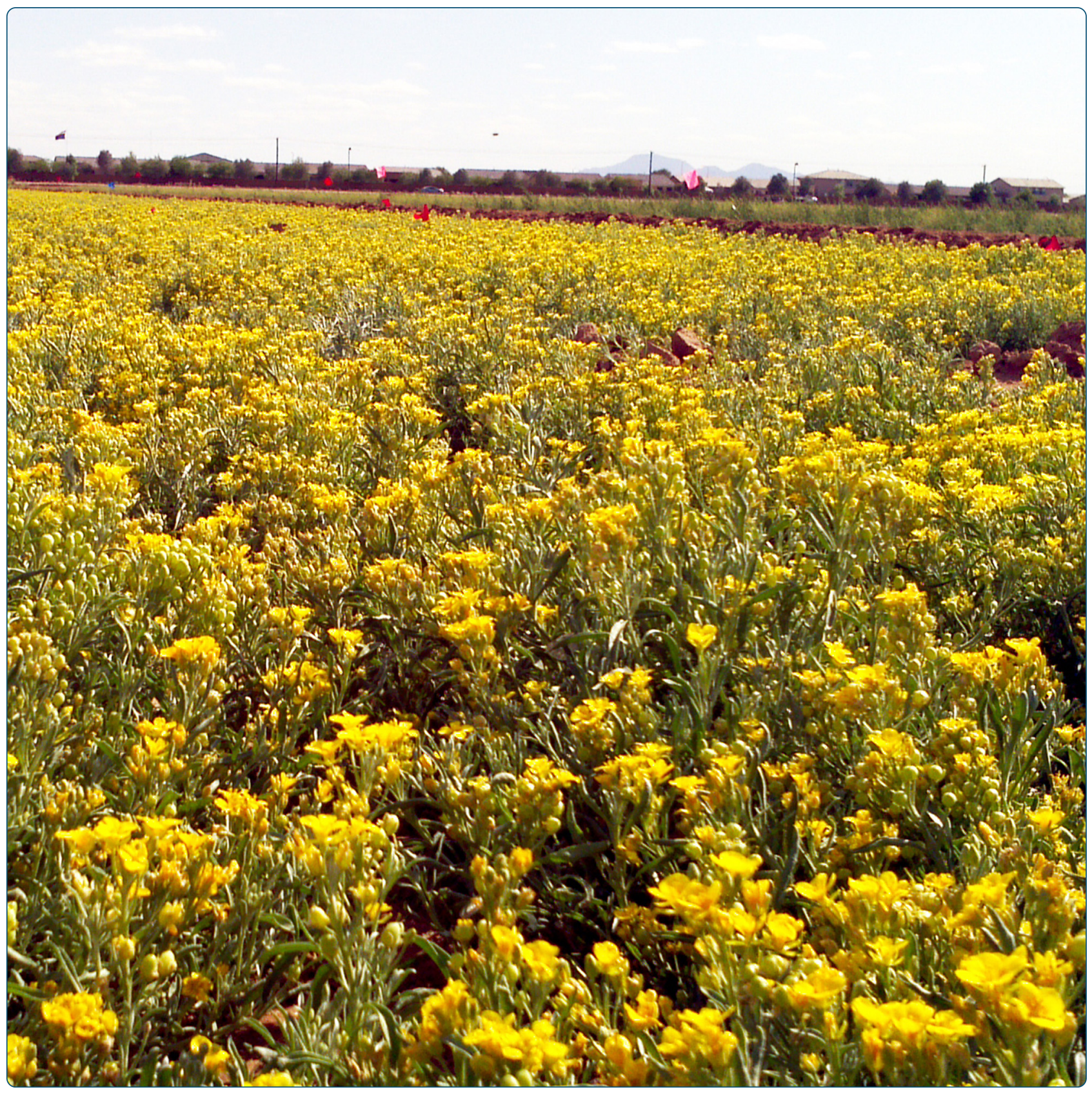

Identification of hydroxy fatty acid and triacylglycerol metabolism-related genes in lesquerella through seed transcriptome analysis

Kim and Chen 


\title{
Identification of hydroxy fatty acid and triacylglycerol metabolism-related genes in lesquerella through seed transcriptome analysis
}

Hyun Uk Kim ${ }^{1 *}$ and Grace Qianhong Chen ${ }^{2 *}$

\begin{abstract}
Background: Castor oil is the only commercial source of hydroxy fatty acid that has industrial value. The production of castor oil is hampered by the presence of the toxin ricin in its seed. Lesquerella seed also accumulates hydroxy fatty acid and is free of ricin, and thus it is being developed as a new crop for hydroxy fatty acid production. A high-throughput, large-scale sequencing of transcripts from developing lesquerella seeds was carried out by 454 pyrosequencing to generate a database for quality improvement of seed oil and other agronomic traits. Deep mining and characterization of acyl-lipid genes were conducted to uncover candidate genes for further studies of mechanisms underlying hydroxy fatty acid and seed oil synthesis.

Results: A total of 651 megabases of raw sequences from an mRNA sample of developing seeds was acquired. Bioinformatic analysis of these sequences revealed 59,914 transcripts representing 26,995 unique genes that include nearly all known seed expressed genes. Based on sequence similarity with known plant proteins, about $74 \%(19,861)$ genes matched with annotated coding genes. Among them, 95\% $(18,868)$ showed highest sequence homology with Arabidopsis genes, which will allow translation of genomics and genetics findings from Arabidopsis to lesquerella. Using Arabidopsis acyl-lipid genes as queries, we searched the transcriptome assembly and identified 615 lesquerella genes involved in all known pathways of acyl-lipid metabolism. Further deep mining the transcriptome assembly led to identification of almost all lesquerella genes involved in fatty acid and triacylglycerol synthesis. Moreover, we characterized the spatial and temporal expression profiles of 15 key genes using the quantitative PCR assay.

Conclusions: We have built a lesquerella seed transcriptome that provides a valuable reference in addition to the castor database for discovering genes involved in the synthesis of triacylglycerols enriched with hydroxy fatty acids. The information obtained from data mining and gene expression profiling will provide a resource not only for the study of hydroxy fatty acid metabolism, but also for the biotechnological production of hydroxy fatty acids in existing oilseed crops.
\end{abstract}

Keywords: Hydroxy fatty acid, Lesquerella, Physaria fendleri, Seed, Transcriptome, Triacylglycerol, Gene expression, Quantitative polymerase chain reaction

\section{Background}

Lesquerella [Physaria fendleri, formerly Lesquerella fendleri (Gray) Wats.] [1], is a potential Brassicaceae oilseed crop for the southwest region of the United States. The seed oil of lesquerella is rich in lesquerolic acid (14hydroxy-eicos-cis-11-enoic acid: 20:1-OH), a hydroxy

\footnotetext{
* Correspondence: hukim64@korea.kr; grace.chen@ars.usda.gov

${ }^{1}$ Department of Agricultural Biotechnology, National Academy of Agricultural Science, Rural Development Administration, Jeonju 560-500, Republic of Korea ${ }^{2}$ U.S. Department of Agriculture, Western Regional Research Center, Agricultural Research Service, 800 Buchanan Street, Albany, CA 94710, USA
}

fatty acid (HFA) comprising $55-60 \%$ of total seed fatty acids [2-6]. The conventional source of HFA is castor (Ricinus communis) seeds; 90\% of castor oil is ricinoleic acid (12-hydroxy-octadec-cis-9-enoic acid: 18:1-OH). Ricinoleic acid and its derivatives are used as raw materials for numerous industrial products, such as lubricants, plastics and surfactants [7]. The production of castor oil, however, is hampered by the presence of the toxin ricin and hyper-allergic $2 \mathrm{~S}$ albumins in its seed. Lesquerella on the other hand, does not have such biologically toxic compounds, and thus its oil represents a 
safe source of HFA. With the development of clean and renewable energy, hydroxy fatty acid methyl esters of lesquerella oil were found to be excellent lubricity enhancers in diesel fuels $[8,9]$ that replace sulfur-based petroleum lubricity additives, and thus reduce environmental pollution. Besides the HFA, several co-products can be obtained from lesquerella. Seed meal after oil extraction is high in protein and the amino acid lysine and could be used as livestock feed [10,11]. Gums from the seed coat and seed meal could be used as thickening or gelling agents in food and pharmaceutical products [12-15].

Considerable efforts have been made to improve the agronomics of lesquerella through plant breeding [2,16-19]. Furthermore, stable genetic transformation has been established in lesquerella [20], which provides means to quickly improve this crop through genetic engineering. Currently, the United States Department of Agriculture (USDA) National Plant Germplasm System (NPGS) has a Phyasaria germplasm collection of over 212 accessions representing 32 species. Variation in fatty acids among species was reported. In species $P$. lindheimeri and $P$. pallida, 20:1-OH was the most abundant, comprising over $80 \%$ in seed oil [21]. Some species have seeds with oil rich in other HFAs, such as densipolic acid (12-hydroxy-octadec-cis-9,15-enoic acid: 18:2OH) in $P$. perforata, $P$. stonensis, $P$. densipila, $P$. lyrata, and $P$. lescurii (average over 40\%) [21-23]. In species $P$. auriculata and P. densiflora, auricolic acid (14-hydroxyeicos-cis-11,17-enoic acid: $20: 2-\mathrm{OH}$ ) was the prevalent HFA, at $34-40 \%$ levels $[24,25]$. These species with different HFA profiles are valuable genetic resource and may contribute to the improvement of lesquerella cultivars.

Seed oil is stored as triacylglycerol (TAG). Biosynthesis of TAG in lesquerella follows the pathways for fatty acid (FA) in the plastid and TAG in the endoplasmic reticulum (ER) [26,27]. After the FAs are synthesized in the plastid (mostly oleic acid 18:1 with small amounts of palmitic acid 16:0 and stearic acid 18:0), they are released and then converted to acyl-Co-enzyme A (CoA). The newly synthesized acyl-CoAs can be incorporated into TAG through the glycerol-3-phosphate (G3P) pathway also known as the Kennedy pathway $[28,29]$. Briefly, G3P is first acylated by glycerol-3-phosphate acyltransferase (GPAT), followed by a second acylation by the acyl-CoA:acylglycerol-3-phosphate acyltransferase (LPAT), yielding phosphatidic acid (PA). PA is then hydrolyzed to form diacylglycerol (DAG), which is finally used as a substrate for the diacylglycerol acyltranstransferase (DGAT) to produce TAG. The newly synthesized acyl-CoAs can also be incorporated directly into membrane lipid phospatidylcholine (PC) by the acyl editing reactions or Lands cycle [30-32]. These acyl editing reactions can be catalyzed either by forward and reverse reactions of lyso-
PC acyltransferase (LPCAT) to yield acyl-CoA, or by a phospholipase A-type activity to yield a free FA that then is activated to acyl-CoA. Since PC is the substrate for many FA-modifying enzymes (desaturase, hydroxylase, etc.), rapid de-acylation and re-acylation of PC results in an acyl-CoA pool enriched with modified FAs, which are then utilized for TAG synthesis [33,34]. Additionally, accumulating evidence indicates that many plants utilize PC-derived DAG to synthesize TAG. The main PC to DAG conversion is catalyzed by phosphatidylcholine:diacylglycerol cholinephosphotransferase (PDCT) through the phosphocholine headgroup exchange between $\mathrm{PC}$ and DAG $[35,36]$. Thus acyl editing and PC-DAG interconversion through LPCAT and PDCT, respectively, may co-contribute to the formation of TAGs with enriched modified FAs. Besides, TAG synthesis is not as simple as the sequential acylation of glycerol with GPAT, LPAT, and DGAT by the Kennedy pathway. The enzyme Phospholipid:DAG acyltransferase (PDAT) also syntheses TAG by transacylation of the $s n-2$ FA from PC onto $s n-3$ position of DAG, with lyso-PC as a co-product [37].

The molecular and biochemical bases of HFA synthesis have been investigated mostly in castor, lesquerella, and Arabidopsis (review) [38]. Based on studies in castor, $18: 1-\mathrm{OH}$ is formed by the hydroxylation of $18: 1$ esterified to the $s n-2$ position of PC $[39,40]$. Then, the $18: 1-\mathrm{OH}$ is released from $18: 1-\mathrm{OH}-\mathrm{PC}$ and activated to 18:1-OH-CoA. In lesquerella, due to an efficient microsomal elongation system, newly formed $18: 1-\mathrm{OH}-\mathrm{CoA}$ is elongated to 20:1-OH-CoA [24,25,41]. Genes encoding the oleate 12-hydroxylase $(F A H)$ have been isolated from castor (RcFAH12) [42] and lesquerella (PfFAH12) [43]. (LFAH12 or LfFAH12 were used in publications before this report). Arabidopsis is a model oilseed that usually does not produce HFA. Expression of the RcFAH12 in Arabidopsis leading to HFA accumulation thus demonstrated that this enzyme is directly responsible for synthesis of 18:1-OH [42,44]. Expression of PfFAH12 in Arabidopsis $[43,44]$ and yeast [45] has revealed that the lesquerella enzyme is bifunctional and can catalyze $\Delta 12$ hydroxylation to produce $18: 1-\mathrm{OH}$ and $\Delta 12$ desaturation to produce 18:2. In lesquerella, a gene encoding a condensing enzyme, PfKCS18 (LfKCS3 was used in publications before this report) has been isolated, and its activity has been shown to specifically catalyze elongation of $18: 1-$ OH-CoA [41]. Besides 18:1-OH and 20:1-OH, lesquerella seed accumulates a low level of 20:2-OH, which is formed by a microsomal $\Delta 15$ desaturase $[24,25,44]$.

Although enzymatic reactions and key genes involved in the HFA synthesis have been elucidated, mechanisms contributing to the accumulation of HFA in TAG are largely unknown. Transgenic experiments have consistently failed to achieve high yields of desired HFAs. Seedspecific expression of RcFAH12 in Arabidopsis resulted 
in HFA accumulation at $17 \%$ of total seed lipids [44,46-48], which is much lower than $90 \%$ level of $18: 1$ $\mathrm{OH}$ in castor seeds. Efforts have been made to search for additional genes, especially those involved in a final step of TAG synthesis. It was shown that co-expression of a second gene, RcDGAT2 [49] or a RcPDAT [50,51] boosted HFA content from $17 \%$ to nearly $30 \%$ or 25 $27 \%$, respectively. When triple transgenic Arabidopsis (carrying RcFAH, RcDGAT2 and RcPDAT1A) is compared with a double transgenic line (carrying RcFAH and RcPDAT1A), HFA increased slightly from $25.4 \%$ to $26.7 \%$ [50,51]. With the discovery of PDCT, a castor gene $R c P D C T$ was co-expressed in the transgenic Arabidopsis line carrying RcFAH. It indeed increased HFA from $17 \%$ to $23 \%$ in Arabidopsis [35]. Additional expression of RcDGAT2 further enhanced the HFA content to $28 \%$ [35].

Broadening our knowledge on HFA-containing TAG biosynthesis undoubtedly requires the identification of more genes involved in HFA and TAG metabolism. The high-throughput 454 GX FLX pyrosequencing is a superior technology for transriptome analysis. It revolutionizes science by enabling users to acquire massive genome-wide data rapidly with low cost and labor. Because the method increases sequencing depth and coverage, it allows assembly of overlapping reads without a references sequence. It is particularly suitable for use in organisms whose genomic sequences are unknown. Prior to our work, there are only 71 lesquerella microsatellite sequences and ESTs in GenBank (http:// www.ncbi.nlm.nih.gov/nucest/). In this study, we adopted 454 GX FLX pyrosequencing to analyze the seed transcriptome of lesquerella. We describe here identification of 26,995 unique transcripts from a total of 651 mega-base raw sequences, including transcripts for the majority of enzymes involved in lipid biosynthesis and metabolism. We further characterize the expression profiles of 15 key lipid genes in various tissues of lesquerella, including developing seeds, leaf, stem, root, and flower buds using quantitative PCR (qPCR) assays. Our results provide information on key target genes that can be useful in the design of future studies involving manipulation of HFA production in plants.

\section{Results and discussion}

The transcriptome represents a major source for lesquerella seed genes

To obtain a comprehensive profile of the transcriptome of lesquerella seeds, selecting a sample at an optimal stage during seed development is critical. According to our previous studies in lesquerella, the entire course of seed development took about 49 days after pollination (DAP) [52]. When developing seeds entered midmaturation stages, 28-35 DAP, storage lipids, proteins, and other components of dry weights accumulated at maximum rates. Based on analysis of HFA accumulation and gene expression [5], we observed rapid synthesis and accumulation of TAG and major HFA (20:1-OH) from 28 to 35 DAP. Accompanying the accumulation of 20:1-OH, transcript levels of hydroxylase gene, PfFAH12, and elongase gene, PfKCS18, also increased steadily [5]. The collective data suggest that seeds at 28-35 DAP are enriched in the transcripts for enzymes involved in the synthesis and accumulation of 20:1-OH. Therefore, we determined that $30 \mathrm{DAP}$ is a suitable time point for seed transcriptomic analyses. By twice deep sequencing using a cDNA library prepared from developing lesquerella seeds at 30 DAP, we generated a total of 1,568,943 clean reads which is equivalent to $651,314,783$ bases (Table 1 ). Because there is no reference genome of lesquerella, de novo assembly was performed by GS De Novo Assembler (v 2.6). Among total reads, 496,246 reads were completely assembled and 109,151 reads were partially assembled to generate total 38,002 isotigs (Table 2). The average isotig length is $988 \mathrm{bp}$ (Table 2), longer than the average isotig size of 828 bp observed in bitter melon seed transcriptome using Cap3 assembly software [53], 744 bp in Ammopiptanthus monolicus root transcriptome using Newbler [54], and 697 bp in Camelina sativa seed transcriptome using Trinity [55]. A mean isotig length of $1492 \pm 899$ bp was reported for camelina seed transcriptome [56]. However, this number was obtained by assembling 454 reads (mean length, $373 \pm 129 \mathrm{bp}$ ) together with Sanger sequenced EST clones (mean length, $555 \pm 169$ bp) using NEWBLER v2.3 GS Assembler. About 35,573 reads not overlapping with any other reads were assembled to 21,912 singletons (Tables 1 and 2). Since our singletons were cleaned and validated with SeqClean and Lucy, the numbers of singletons and isotigs in combination indicate 59,914 total protein coding transcripts in our seed transcriptome. Overall, isotigs and singletons together were assembled from 640,970 reads which counted $41 \%$ of total reads (Table 1). About $57 \%$ of reads were identified as repeated regions (Table 1). A total of 901,748 repeat region reads were blast searched in SILVA rRNA database [57]. Among

\section{Table 1 Summary of sequencing reads}

\begin{tabular}{ll}
\hline Number of reads & $1,568,943$ \\
Number of bases & $651,314,783$ \\
Assembled reads & 496,246 \\
Partially assembled reads & 109,151 \\
Singleton & 35,573 \\
Repeat & 901,748 \\
Outlier+ & 15,725 \\
Reads too short to assemble & 10,500
\end{tabular}

+The read was identified by the GS De Novo Assembler as problematic. 
Table 2 Summary of de novo assembly

\begin{tabular}{llll}
\hline & Isogroups & Isotigs & Singletons \\
\hline Number & 26,995 & 38,002 & 21,912 \\
Average contig count & 1.7 & 2.132 & \\
Largest contig count & 2,257 & 16 & \\
Number with one contig & 22,649 & 22,994 & \\
Average isotig count & 1.4 & & \\
Largest isotig count & 97 & & \\
Number with one isotig & 22,698 & & \\
Number of bases (nt) & & $37,527,552$ & \\
Average isotig size (bp) & & 987.515 & \\
N50 isotig size (bp) & & 1,310 \\
Largest isotig size (bp) & & 11,074 & \\
\hline
\end{tabular}

The number of valid singleton after SeqClean and Lucy.

"The half size of all bases reside in isotigs.

them, 863,953 reads (96\%) matched rRNA sequences and 37,795 reads $(4 \%)$ had no homologies. The rRNA sequences encode $28 \mathrm{~S}, 26 \mathrm{~S}, 18 \mathrm{~S}$, and $16 \mathrm{~S}$ ribosomal RNAs. These rRNAs were probably carried over during mRNA purification process. The assembly analysis also generated 26,995 isogroups (Table 2), which represent 26,995 unique genes in the lesquerella seed transcriptome (Table 2).

To functionally categorize lesquerella isotigs and singletons, we performed Gene ontology (GO) analysis. The isotigs and singletons were searched against NCBI non-redundant protein database (NR), using the Blastx program with an E-value cut-off of $1 \mathrm{e}^{-3}$. Among 38,002 isotigs and 21,912 singletons, 33,313 isotigs (88\%) and 11,247 singletons (51\%) had at least one match to known protein sequences in the NCBI NR database. These isotigs or singletons fell mainly into three GO categories: biological process $(11,977,32 \%$ for isotigs; $4,641,21 \%$ for singletons), cellular component $(9,648,25 \%$ for isotigs; $2,850,13 \%$ for singletons), and molecular function (11,687, $31 \%$ for isotigs; $3,756,17 \%$ for singletons). The remaining 4,689 (12\%) isotigs and 10,663 (49\%) singletons were not assigned (Figures 1A, 2A). In the category of "biological process", a substantial percent of isotigs (22.8\%) (Figure 1B) and singletons (18.8\%) (Figure 2B) were classified in sub-category 'metabolic process'. In category "cellular component", 'cell part' had a largest percent of isotigs (26.4\%) (Figure 1B) and singletons (28.5\%) (Figure 2B). Within the category of "molecular function", 'catalytic activity' and 'binding' are the two top sub-categories, with $34.9 \%$ and $34.0 \%$ respectively for isotigs, (Figure 1B), and $28.4 \%$ and $29 \%$ respectively for singletons (Figure 2B). As indicated above, the assemble analysis also generated 26,995 isogroups or unique genes. When these unique genes were searched against NCBI NR database, 19,861 isogroups (74\%) matched annotated coding genes, whereas 3,134 found no homologous sequences. Besides, about 18,868 (95\%) of the 19,861 isogroups with matches to known protein coding sequences had the highest homology to genes from Arabidopsis, a model dicot plant. Table 3 lists the top 50 most highly expressed genes represented by isogroups in the lesquerella seed transcriptome and their corresponding gene products based Arabidopsis orthologs. Among them, genes involved in lipid biosynthesis and metabolism were detected. These included genes encoding fatty acid desaturase 3 (FAD3), 3-ketoacyl-CoA synthase (PfKCS18), acyl-activating enzyme 17 (AAE17), hydroxysteroid dehydrogenase 5 (HSD5) and oleosins. Overall, these results demonstrate that the transcriptome data presented here provide comprehensive representation of expressed genes in developing lesquerella seed. These annotations provide a major new resource for investigating specific processes, structures, functions, and pathways that will guide research on lesquerella. Moreover, the transcriptome analysis indicates that lesquerella is closely related to Arabidopsis, which allows for translational research for seed quality enhancement in lesquerella.

\section{The transcriptome covers a broad spectrum of genes involved in acyl-lipid metabolism}

Since lesquerella is a Brassicaceae oilseed crop, we used the acyl-lipid metabolism database [58] developed for model Brassicaceae oilseed Arabidopsis [31] to investigate lesquerella genes involved in acyl-lipid metabolism. We queried the transcriptome assembly to predict lesquerella orthologs of Arabidopsis lipid metabolic pathway components using reciprocal best-hits $(\mathrm{RBH})$ blast approach. A total of 1,066 Arabidopsis genes from 16 sub acyl-lipid metabolism group from the lipid metabolism database [58] were used to query the lesquerella transcriptome using local blastN. Although our lesquerella genes were limited to developing seed, whereas the acyl-lipid metabolism database includes whole genomewide acyl-lipid genes, we are able to detect total 615 lesquerella genes representing each sub acyl-lipid metabolism group (Table 4). Two subgroups, Fatty acid synthesis and Triacylglycerol biosynthesis, of lesquerella genes had 29 and 59 members, respectively, which are very close to the numbers of 28 and 69 respectively of Arabidopsis genes obtained from a microarray study on genes expressed in seeds [59]. A total of 1,989 isotigs and singletons involved in acyl-lipid metabolism showing high sequence identity with Arabidopsis genes were identified. The average number of transcripts for each subgroup were: 8 in subgroup of triacylglycerol biosynthesis and 4 in subgroup of fatty acid synthesis. The rest of subgroups each had 2-3 average transcript numbers. The result indicates that our seed transcriptome is 


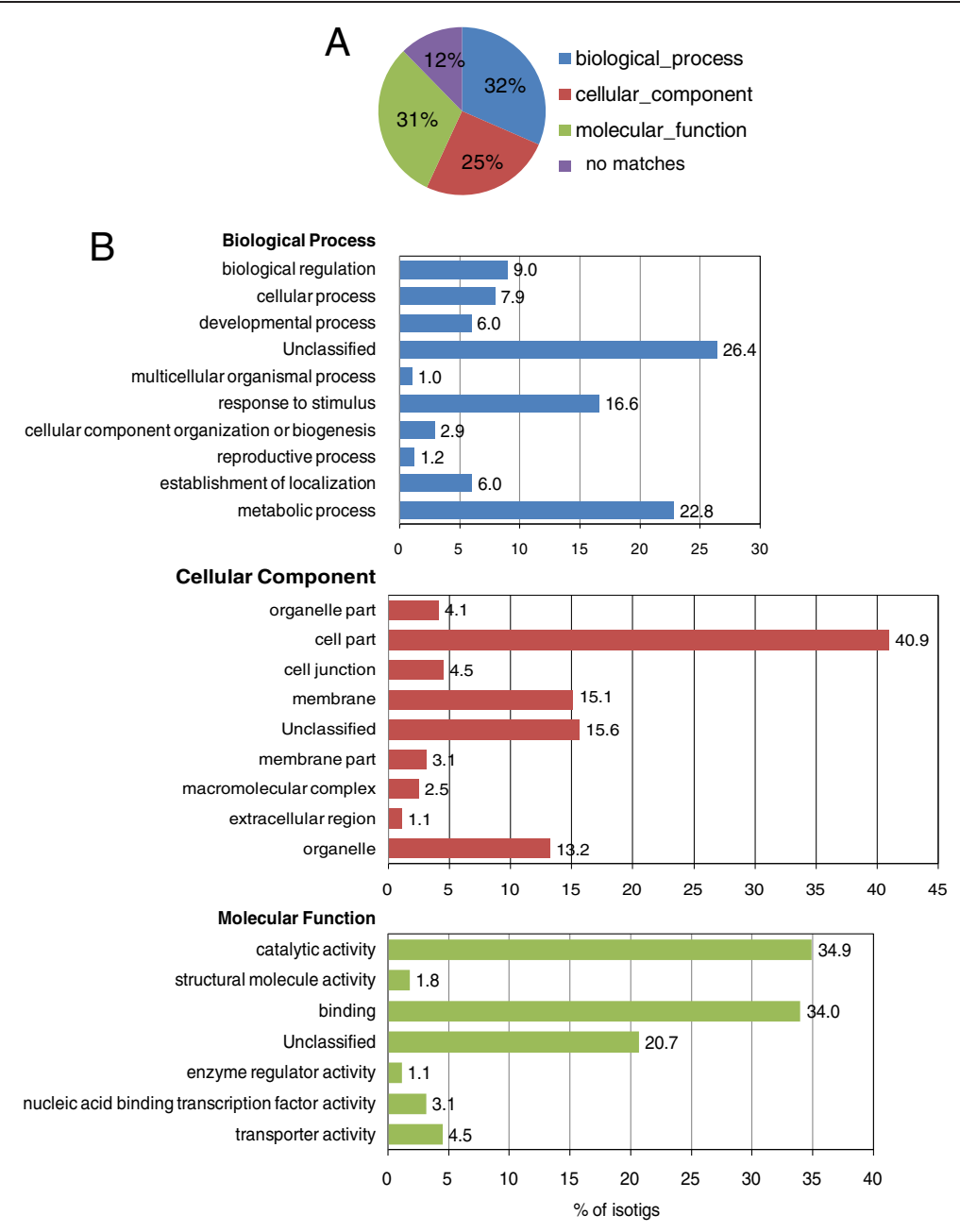

Figure 1 Gene ontology (GO) annotation of all detected isotigs. (A) Overall distribution of 38,002 isotigs into major GO categories. (B) The histogram shows the percentage ( $x$ axis) of isotigs within functional subdivisions ( $y$ axis) of each of the three major GO categories.

highly representative of transcripts for fatty acid and TAG biosynthesis. We conclude that our selection of developing seeds at 30 DAP was suitable for studying acyllipid metabolism in lesquerella.

\section{Genes involved in fatty acid and TAG biosynthesis are well-represented in the transcriptome}

To understand hydroxy fatty acid biosynthesis and metabolism in lesquerella, we further deeply mined the transcriptome assembly for genes involved in fatty acid and TAG biosynthesis. Using known Arabidopsis genes listed in the acyl-lipid metabolism database, we identified lesquerella orthologs for nearly all the genes involved in fatty acid and TAG biosynthesis; the number of isotigs for each gene varied from 1 to 58 (Table 5, Figure 3). Among 32 genes, 20 (63\%) had a representative isotig encoding full length cDNA. The ones coding for partial cDNA sequences covered $23-97 \%$ of their full length sequences. Strikingly, we found a high percentage of sequence identity between Arabidopsis and lesquerella genes. Out of 32 genes, 20 had nucleotide identity at 90$99 \%$, with an average identity overall of $90 \%$. The remaining 12 had $80-89 \%$ identity (Table 5). The very high sequence identity between Arabidopsis and lesquerella genes suggests a similar high degree of conservation of their functions in seeds that enables translational research and facilitates genetic engineering of lesquerella lines with desirable oil content and fatty acid composition.

\section{Fatty acid biosynthesis in plastids}

We examined our collection of lesquerella transcripts for representatives of key genes encoding the known steps of fatty acid biosynthesis in plastids (Figure 3). Fatty acid biosynthesis begins with the rate-limiting conversion of acetyl-CoA to malonyl-CoA by acetyl-CoA carboxylase, a heteromeric complex enzyme composed of 4 subunits: 1 beta-carboxyltransferase $(\beta-C T)$ encoded by the plastid genome and biotin carboxyl-carrier 


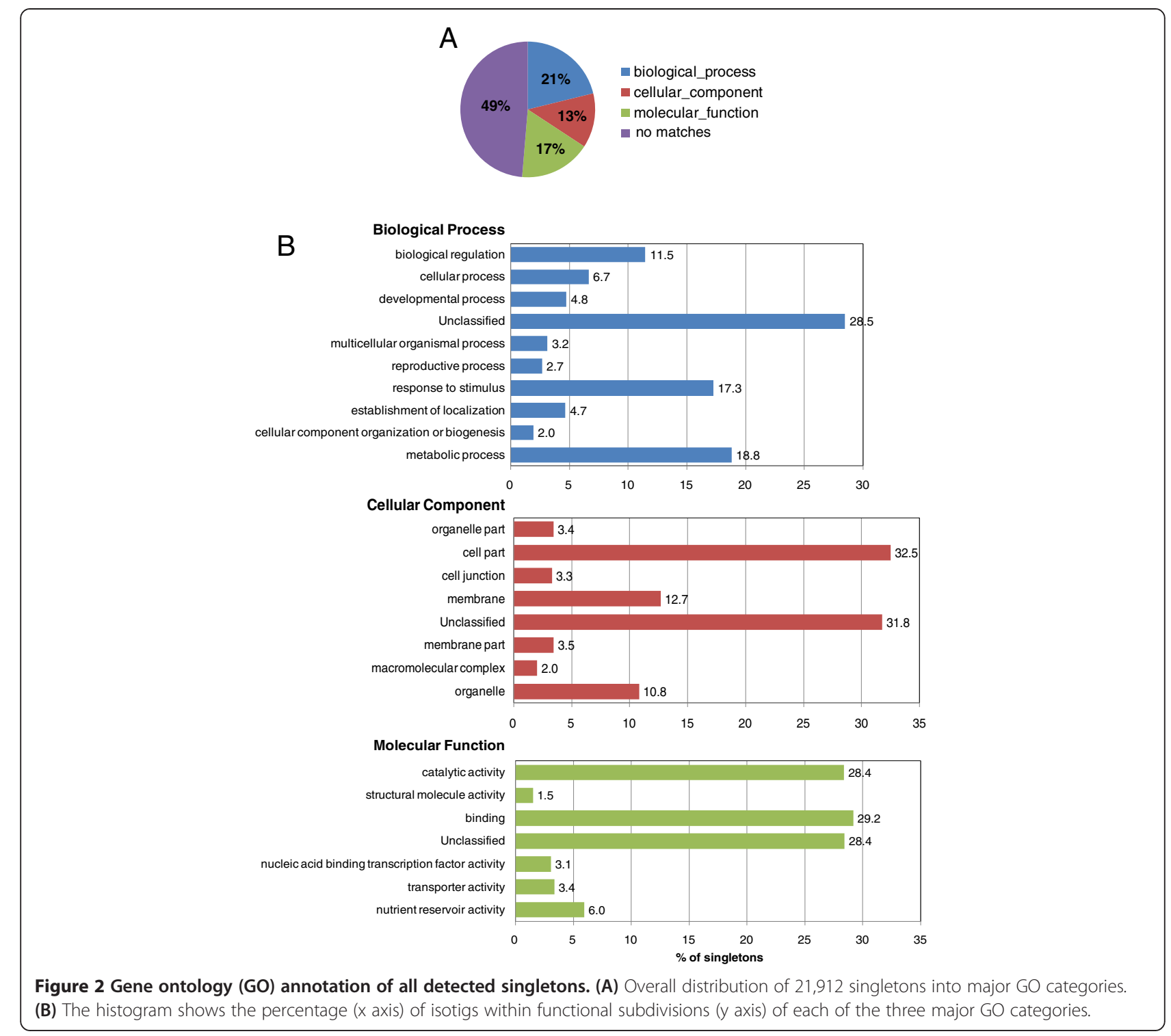

protein (BCCP), biotic carboxylase $(\mathrm{BC})$, and alphacarboxyltransferase $(\alpha-C T)$, each encoded by the nuclear genome. In the lesquerella transcriptome, transcripts encoding $\mathrm{BCCP}$ and $\alpha-\mathrm{CT}$ subunits were identified with 10 isotigs and 17 isotigs, respectively (Table 5). In Arabidopsis, two paralogous of $B C C P$ genes, $B C C P 1$ (At5g16390) and BCCP2 (At5g15530) were characterized with $B C C P 1$ being the more highly expressed during embryo development [60]. In lesquerella seed, 7 isotigs of PfBCCP1 and 3 isotigs of PfBCCP2 were identified, and the longest, isotig19563 and isotig07553, shared $88 \%$ and $89 \%$ nucleotide sequence identity, respectively, to Arabidopsis homologous. We did not detect $B C$ and $\beta$ $C T$ in the lesquerella seed transcriptome, which could be due to a low level of their transcripts in 30 DAP seeds. We detected lesquerella homologs of all five isoforms of plastid acyl-carrier proteins (ACP) reported in
Arabidopsis (At3g05020, At1g54580, At1g54630, At4g25050, At5g27200) [61]. Among them, PfACP5 (6 isotigs), corresponding to At5g27200 isoform, was the mostly expressed (Table 5). A gene encoding malonylCoA ACP transferase (MCMT) was also identified in lesquerella with 5 isotigs, and the longest isotig 17972 showed 93\% nucleotide sequence identify with its Arabidopsis MCMT homolog (At2g30200).

Fatty acid synthesis is continued by an acyl-chain specific condensing enzyme subunit (KASIII, I, and II), and the common component of 3-ketoacyl-ACP reductase (KAR), 3-hydroxyacyl-ACP dehydratase (HAD), and 2-enoyl-ACP reductase (ER) (Figure 3 ). We tried to identify transcripts for three key fatty acid synthases, 3ketoacyl-ACP synthase (KAS) III, I, II using Arabidopsis KASIII (At1g62640), KASI (At5g46290) and KASII (At1g74960) genes as queries, but only PfKASII was 
Table $\mathbf{3}$ List of gene products for the $\mathbf{5 0}$ most abundant isotigs

\begin{tabular}{|c|c|c|c|}
\hline Isogroup & \# of istotigs & Gene products & Gene ID \\
\hline isogroup00026 & 97 & HSD5 (hydroxysteroid dehydrogenase 5) & AT4G10020 \\
\hline isogroup00055 & 64 & ATAILP1 & AT5G19140 \\
\hline isogroup 00045 & 55 & OLEOSIN 1 & AT4G25140 \\
\hline isogroup00062 & 49 & GAMMA-VPE, vacuolar-processing & AT4G32940 \\
\hline isogroup00056 & 46 & FDH (formate dehydrogenase) & AT5G14780 \\
\hline isogroup00010 & 44 & FAD3 & AT2G29980 \\
\hline isogroup 00025 & 41 & Physaria fendleri 3-ketoacyl-CoA synthase & AAK62348.1 \\
\hline isogroup00041 & 37 & PRXR1 & AT4G21960 \\
\hline isogroup00046 & 36 & HSC70-1 & AT5G02500 \\
\hline isogroup00048 & 36 & OLEOSIN 4 & AT3G27660 \\
\hline isogroup00071 & 36 & unknown protein & AT1G62220 \\
\hline isogroup00037 & 35 & CESA3 (cellulose synthase 3) & AT5G05170 \\
\hline isogroup00047 & 35 & Class-II DAHP synthetase family & AT1G22410 \\
\hline isogroup00072 & 35 & UAP56B (homolog of human UAP56 B) & AT5G11200 \\
\hline isogroup 00073 & 35 & UBQ1 (ubiquitin extension protein 1) & AT3G52590 \\
\hline isogroup 00042 & 34 & ATNTT2 & AT1G15500 \\
\hline isogroup00079 & 34 & splicing factor PWI domain-containing protein & AT1G60200 \\
\hline isogroup00001 & 32 & $12 \mathrm{~S}$ seed storage protein $\mathrm{CRU} 4$ & AT5G44120 \\
\hline isogroup00049 & 32 & FDM1 (factor of DNA methylation 1) & AT1G15910 \\
\hline isogroup 00050 & 32 & XYP1 (xylogen protein 1) & AT5G64080 \\
\hline isogroup 00043 & 31 & HAl1 (highly ABA-induced PP2C gene 1) & AT5G59220 \\
\hline isogroup00063 & 31 & RING-type Zinc finger protein & AT5G25560 \\
\hline isogroup00023 & 30 & ALPHA-TIP (alpha-tonoplast intrinsic protein) & AT1G73190 \\
\hline isogroup00057 & 30 & ACO2 (ACC oxidase 2) & AT1G62380 \\
\hline isogroup00086 & 30 & CESA1 (cellulose synthase 1) & AT4G32410 \\
\hline isogroup00080 & 26 & BBD2 (bifuctional nuclease in basal defense response 2) & AT1G19660 \\
\hline isogroup00027 & 25 & Heat shock protein $81-2$, & AT5G56030 \\
\hline isogroup00030 & 25 & BGLU37 (beta glucosidase 37) & AT5G25980 \\
\hline isogroup 00044 & 24 & NLP4 (nin-like protein 4) & AT1G20640 \\
\hline isogroup00058 & 24 & TUA6 (tubulin alpha-6) & AT4G14960 \\
\hline isogroup00074 & 23 & Pyruvate kinase family & AT5G08570 \\
\hline isogroup00038 & 22 & UBQ1 (ubiquitin extension protein 1) & AT3G52590 \\
\hline isogroup00087 & 22 & PEPR1 (PEP1 receptor 1) & AT1G73080 \\
\hline isogroup00088 & 22 & Methionine synthesis 1 & AT5G17920 \\
\hline isogroup00033 & 21 & GTP binding Elongation factor Tu family & AT5G60390 \\
\hline isogroup00075 & 21 & TCTP (translationally controlled tumor protein) & AT3G16640 \\
\hline isogroup00081 & 20 & DRP2B (dynamin related protein 2B) & AT1G59610 \\
\hline isogroup00089 & 20 & AOAT2 (alanine-2-oxoglutarate aminotransferase 2) & AT1G70580 \\
\hline Isogroup00011 & 19 & $2 \mathrm{~S}$ seed storage protein 3 & AT4g27160 \\
\hline isogroup00090 & 19 & Papain family cysteine protease & AT4G16190 \\
\hline isogroup00064 & 17 & Phosphoinositide phosphatase family & AT1G17340 \\
\hline isogroup00065 & 17 & ALAAT1 (alanine aminotransferase 1) & AT1G17290 \\
\hline isogroup 00040 & 16 & GTP binding Elongation factor Tu family protein & AT5G60390 \\
\hline isogroup00066 & 16 & Leucine-rich repeat (LRR) family protein & AT5G45510 \\
\hline
\end{tabular}


Table $\mathbf{3}$ List of gene products for the $\mathbf{5 0}$ most abundant isotigs (Continued)

\begin{tabular}{llll}
\hline isogroup00083 & 16 & HSP70 (heat shock protein 70) & AT3G12580 \\
isogroup00067 & 15 & BGLU37 ( beta glucosidase 37) , & AT5G25980 \\
isogroup00068 & 15 & GTP binding Elongation factor Tu family & AT5G60390 \\
isogroup00078 & 15 & F-box/RNI-like superfamily protein & AT4G15475 \\
isogroup00053 & 14 & Copper amine oxidase family & AT4G12290 \\
isogroup00061 & 13 & AAE17(acyl-activating enzyme 17) & AT5G23050 \\
\hline
\end{tabular}

detected. It had 6 isotigs, and the longest, isotig15566, showed 91\% identity with Arabidopsis seed homolog KASII [62]. The other two KAS transcripts are apparently missing or rare in the 30-day seed transcriptome. Based on Arabidopsis KAR (At1g24360), HAD (At5g10160), and ER (At2g05990), we detected PfKAR (5 isotigs) , PfHAD (9 isotigs) and PfER (4 isotigs) in lesquerella seed. Stearoyl-ACP desaturase (SAD) catalyzes the conversion of 18:0-ACP to $18: 1-\mathrm{ACP}$ in plastids (Figure 3). Arabidopsis has seven SAD family genes including FAB2 (At2g43710) and DES5 (At1g02630); FAB2 is the most highly expressed [63]. Indeed the FAB2 plays a major role in the reaction [64]. In the seed transcriptome, lesquerella FAB2 homologues were detected with 6 isotigs; No homologous isotig was detected for DES5 (Table 5, Figure 3). Two fatty acid thioesteases, FatA (homologue of At3g25110) and FatB (homologue of At1g08510), were detected with 2 and 4 isotigs, respectively in lesquerella seed (Table 5, Figure 3). Long chain
acyl-CoA synthase (LACS) is located the membrane of plastid outer envelopes and catalyzes addition of CoA to free fatty acids to produce the fatty acyl-CoA's utilized in the endoplasmic reticulum. Two Arabidopsis plastidlocalized LACS9 (At1g77590) and ER-localized LACS8 (At2g04350) have been reported [65,66]. In the lesquerella seed transcriptome, 1 isotig of PfLACS8 and 2 isotigs of PfLACS9 were identified (Table 5, Figure 3).

\section{Endoplasmic reticulum-associated fatty acid hydroxylase, desaturases and elongase}

Seed oil of lesquerella contains 55-60\% 20:1-OH, and two key genes, PfFHA12 and PfKSC18, directly responsible for synthesis of this unusual fatty acid have been previously identified $[41,43]$. In our seed transcriptome, we found 7 and 28 isotigs representing PfFAH12 and PfKCS18, respectively (Table 5, Figure 3 ). The detailed temporal expression patterns of PfFAH12 and PfKCS18 during lesquerella seed development were reported [5]. Both of

Table 4 Number of genes and transcripts involved in acyl-lipid metabolism

\begin{tabular}{|c|c|c|c|c|}
\hline Acyl-lipid metabolism & \#of At genes* & $\begin{array}{l}\text { \#of expressed Pf } \\
\text { genes in seed }\end{array}$ & $\begin{array}{l}\text { \#of detected Pf transcripts } \\
\text { (isotigs and singletons) }\end{array}$ & $\begin{array}{l}\text { \#of transcripts } \\
\text { per Pf gene }\end{array}$ \\
\hline Fatty acid synthesis & 43 & 29 & 106 & 4 \\
\hline Fatty acid elongation, Desaturation \& export from plastid & 27 & 22 & 62 & 3 \\
\hline Prokaryotic galactolipid, Sulfolipid, \& Phospholipid synthesis & 61 & 39 & 91 & 2 \\
\hline Eukaryotic galactolipid \& Sulfolipid synthesis & 36 & 27 & 62 & 2 \\
\hline Triacylglycerol biosynthesis & 87 & 59 & 483 & 8 \\
\hline Eukaryotic phospholipid synthesis \& editing & 75 & 53 & 122 & 2 \\
\hline Triacylglycerol \& fatty acid degradation & 61 & 41 & 119 & 3 \\
\hline Fatty acid elongation \& wax biosynthesis & 246 & 102 & 313 & 3 \\
\hline Sphingolipid biosynthesis & 37 & 30 & 67 & 2 \\
\hline Mitochondria fatty acid \& lipoic acid synthesis & 18 & 11 & 33 & 3 \\
\hline Mitochondria phospholipid synthesis & 24 & 17 & 34 & 2 \\
\hline Lipid trafficking & 6 & 5 & 9 & 2 \\
\hline Cutin synthesis \& transport & 112 & 49 & 152 & 3 \\
\hline Suberin synthesis \& transport & 39 & 25 & 57 & 2 \\
\hline Oxylipin metabolism & 69 & 36 & 66 & 2 \\
\hline Phospholipid signaling & 125 & 70 & 213 & 3 \\
\hline Total & 1066 & 615 & 1989 & 3 \\
\hline
\end{tabular}

*Number of genes obtained from Arabidopsis acyl-lipid metabolism database [58]. 
Table 5 List of expressed genes involved in fatty acid and TAG biosynthesis in lesquerella seed

\begin{tabular}{|c|c|c|c|c|c|c|}
\hline & Gene & At ID & isotigs & $\begin{array}{l}\text { \%of nucleotide } \\
\text { identity }\end{array}$ & $\begin{array}{l}\text { \#of } \\
\text { isotigs }\end{array}$ & $\begin{array}{l}\text { ORF } \\
\text { Partial }(\mathrm{P}) \text { or Full length }(\mathrm{F})\end{array}$ \\
\hline \multirow[t]{15}{*}{ De novo fatty acid biosynthesis and export from plastid } & $B C C P 1$ & At5g 16390 & isotig19563 & 88 & 7 & $P$ \\
\hline & $B C C P 2$ & At5g 15530 & isotig07553 & 89 & 3 & $\mathrm{~F}$ \\
\hline & $a-C T$ & At2g38040 & isotig03259 & 90 & 17 & $\mathrm{~F}$ \\
\hline & MCMT & At2g30200 & isotig17972 & 93 & 5 & $P$ \\
\hline & ACP5 & At5g27200 & isotig07684 & 89 & 6 & $\mathrm{~F}$ \\
\hline & KASII & At1g74960 & isotig15566 & 91 & 6 & $\mathrm{~F}$ \\
\hline & KAR & At1g24360 & isotig08993 & 91 & 5 & $\mathrm{~F}$ \\
\hline & $H A D$ & At5g 10160 & isotig23452 & 88 & 9 & $P$ \\
\hline & $E R$ & At2g05990 & isotig17121 & 93 & 4 & $\mathrm{~F}$ \\
\hline & FAB2 & At2g43710 & isotig11458 & 91 & 6 & $\mathrm{~F}$ \\
\hline & FatA-1 & At3g25110 & isotig17885 & 89 & 1 & $\mathrm{~F}$ \\
\hline & FatA-2 & At3g25110 & isotig17382 & 90 & 1 & $\mathrm{~F}$ \\
\hline & FatB & At1g08510 & isotig09645 & 88 & 4 & $P$ \\
\hline & LACS8 & At2g04350 & isotig15424 & 90 & 1 & $\mathrm{~F}$ \\
\hline & LACS9 & At1g77590 & isotig15369 & 89 & 1 & $P$ \\
\hline \multirow[t]{5}{*}{ Endoplasmic reticulum-hydroxylase, desaturase, and elongase } & FAH12 & AF016103 & isotig27455 & 95 & 7 & $\mathrm{~F}$ \\
\hline & FAD2 & At3g12120 & isotig17487 & 88 & 2 & $\mathrm{~F}$ \\
\hline & FAD3-1 & At2g29980 & isotig17127 & 92 & 1 & $\mathrm{~F}$ \\
\hline & FAD3-2 & At2g29980 & isotig00061 & 84 & 58 & $\mathrm{~F}$ \\
\hline & KCS18 & AF367052 & isotig02742 & 97 & 28 & $\mathrm{~F}$ \\
\hline \multirow[t]{6}{*}{ Acyl-CoA- dependent TAG synthesis in Kennedy pathway } & GPAT9 & At5g60620 & isotig18564 & 94 & 2 & P \\
\hline & LPAT2 & At3g57650 & isotig03872 & 92 & 2 & $\mathrm{~F}$ \\
\hline & DGAT1-1 & At2g19450 & isotig11157 & 91 & 2 & $P$ \\
\hline & DGAT1-2 & At2g19450 & isotig11156 & 90 & 1 & $P$ \\
\hline & DGAT2 & At3g51520 & isotig19956 & 87 & 1 & $\mathrm{~F}$ \\
\hline & DGAT3 & At1g48300 & isotig08903 & 91 & 3 & $\mathrm{~F}$ \\
\hline \multirow[t]{6}{*}{ PC-mediated TAG synthesis } & LPCAT1 & At1g12640 & isotig03769 & 91 & 10 & $\mathrm{~F}$ \\
\hline & LPCAT2 & At1g63050 & isotig16468 & 92 & 1 & $P$ \\
\hline & PDAT1-1 & At5g 13640 & isotig08780 & 92 & 1 & $\mathrm{~F}$ \\
\hline & PDAT1-2 & At5g13640 & isotig08781 & 90 & 15 & $P$ \\
\hline & PDAT2 & At3g44830 & isotig02595 & 86 & 12 & $P$ \\
\hline & PDCT & At3g15820 & isotig25038 & 89 & 1 & $P$ \\
\hline
\end{tabular}




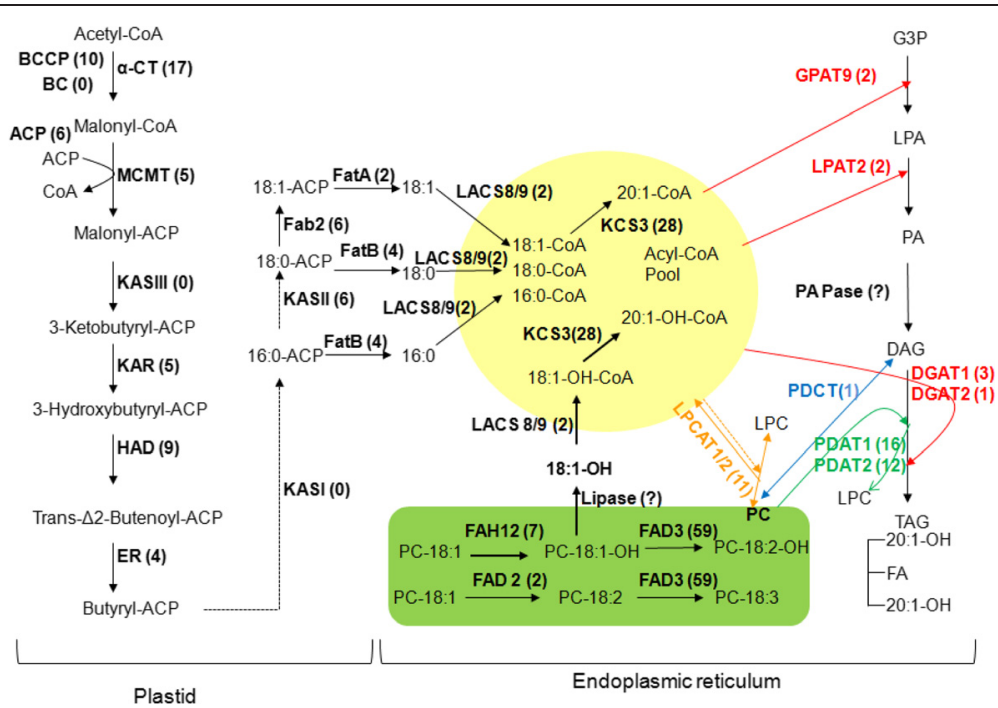

Figure 3 Predicted fatty acid and TAG biosynthetic pathways in P. fendleri seeds. Numbers after the gene are the numbers of isotigs for the corresponding genes in the 454 analysis of the seed cDNA library. The dashed arrows in the plastid fatty acid biosynthetic pathway indicate one or more cycles of acyl-chain elongation that is initiated by 3-ketoacyl-ACP synthase (KAS) I or II. The yellow circle delineates reactions in acyl-CoA pools. The bright green rectangle delineates reactions involving desaturation or hydroxylation of PC. The acyl-CoA dependent Kennedy pathway is indicated with red arrows. PC-mediated TAG synthesis pathways are indicated with green (via PDAT), blue (via PDCT) and orange (via LPCAT) arrows. Abbreviations: BCCP, biotin carboxyl carrier protein subunit of acetyl-CoA carboxylase; BC, biotin carboxylase subunit of acetyl-CoA carboxylase; a-CT, a-carboxyltransferase subunit of acetyl-CoA carboxylase; ACP, acyl carrier protein; MCMT, malonly-COA ACP transferase; KAR, 3-ketoacyl-ACP reductase; $H A D$, 3-hydroxyacyl-ACP dyhydratase; ER, 2-enoyl-ACP reductase; FAB2/SAD, stearoyl-ACP desaturase; FatA, acyl-ACP thioesterase A; FatB, acyl-ACP thioesterase B; LACS, long-chain acyl-CoA synthase; FAH12, $\triangle 12$ oleic acid hydroxylase; FAD2, $\triangle 12$ oleic acid desaturase; FAD3, $\triangle 15$ ( $\omega-3)$ linoleic acid desaturase; KCS3, 3-ketoacyl-CoA synthase 3; GPAT9, glycerol 3-phosphate acyltransferase 9; LPAT2, lysophosphatidic acid acyltransferase 2; PAPase: phosphatidic acid phosphatase; LPCAT, lysophosphatidylcholine acyltransferase 1 and 2; DGAT, diacylglycerol acyltransferase1 and 2; PDAT, phospholipid: diacylglycerol acyltransferase; G3P, glycerol-3-phosphate; LPA, lysophosphatidic acid; PA, phosphatidic acid; LPC, lysophosphatidylcholine; PC, phosphatidylcholine; DAG, diacylglycerol; TAG, triacylglycerol.

the genes showed a bell-shaped expression pattern with a peak at 35 DAP. The increased expression of PfFAH12 and PfKCS18 coincided with the increased synthesis and accumulation of HFA-containing TAG during lesquerella seed development [52].

ER-associated microsomal oleoyl PC desaturase encoded by FAD2 is known to introduce a double bond at the $\Delta 12$ position of $18: 1$ on $\mathrm{PC}$ and convert it to linoleic acid (18:2) (Figure 3). During the entire course of seed development, lesquerella accumulates $18: 2$ at 2.5$6.5 \mathrm{~mol} \%$ in TAG with an average of $4.8 \mathrm{~mol} \%$ in TAG [5]. The presence of a constant level of 18:2 in lesquerella seeds indicates that an oleate desaturease activity is maintained at a relatively steady level. In this study, transcripts of PfFAD2 desaturase were detected with 2 isotigs, fewer than those of PfFHA12 and PfKSC18. The results of temporal expression profile analysis indicated that PfFAD2 is expressed at a relatively constant level throughout most stages of seed development up to 42 DAP, but drops about $95 \%$ by the latest stage sampled, 49 DAP (Figure 4A). The temporal expression pattern of PfFAD2 observed in this study is slightly different from that previously reported (LfFen 1) [5], where a bellshaped pattern of PfFAD2 expression was observed that peaked at 35 DAP and decreased at 42 and 49 DAP [5]. We noted in that study that the change of the expression level of PfFAD2 during seed development was moderately dynamic. In young seeds up to 21 DAP, the expression were already $36-48 \%$ of that of 28 DAP. During 28-35 DAP maturation stages, seeds boosted the expression only 2 to 4 -fold [5]. The difference of PfFAD2 expression pattern at 42 DAP in the two studies could be due to variation in seed sample groups. Nonetheless, the constitutive or less dynamic temporal expression patterns of PfFAD2 indicated its housekeeping function for membrane lipid during seed development. Similar temporal patterns were found in some other house-keeping genes involved in seed oil biosynthesis in Arabidopsis [67] and castor [68]. In this study, we further characterized the expression profile of PfFAD2 in leaf, stem, root and flower bud tissues (Figure 4A). We found that leaf had a level similar to that of developing seeds. The result is consistent with previously reported data of Northern blot analysis [43] or reverse transcription PCR [69]. However, a 4.4-fold higher expression of PfFAD2 in flower than leaf was observed (Figure 4A). Since our flower buds contain about $26 \%$ pollen by weight, it is likely that PfFAD 2 

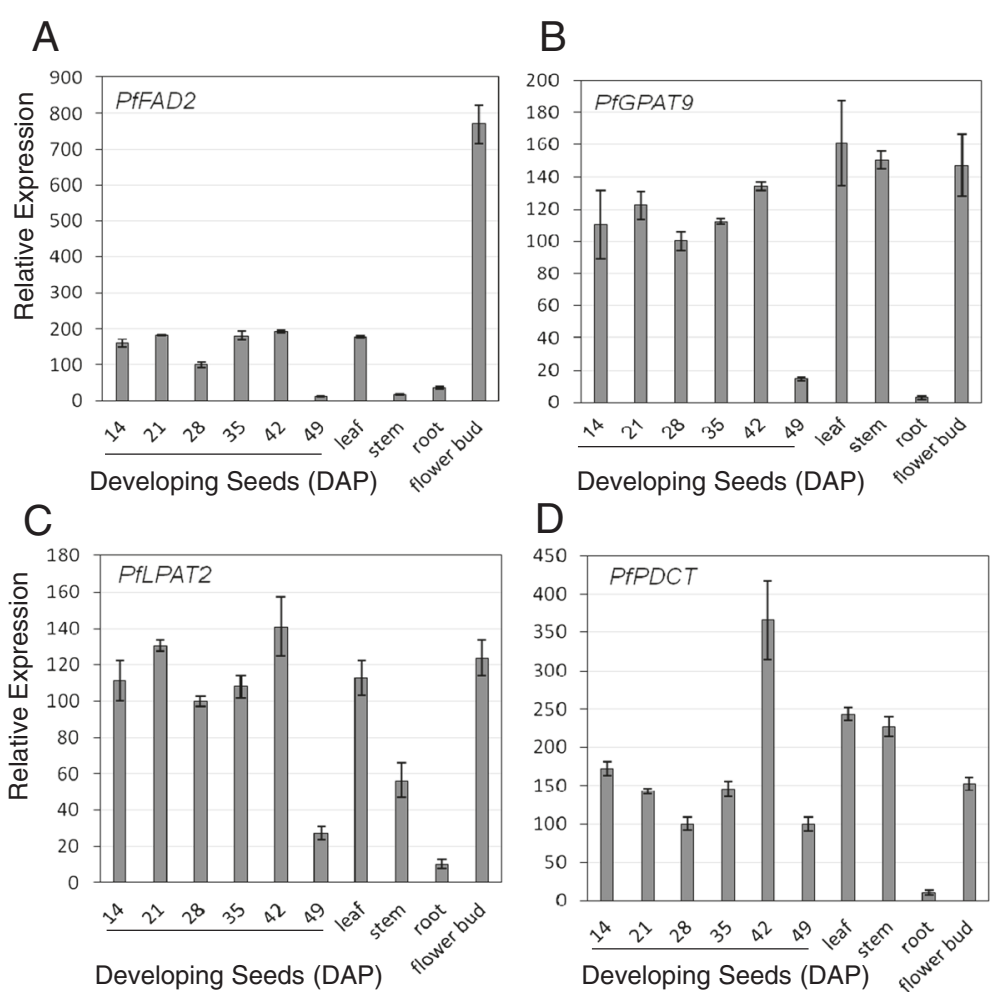

Figure 4 Expression of PfFAD2 (A), PfGPAT9 (B), PfLPAT2 (C) and PfPDCT (D) in major organs and developing seeds determined by qPCR.

Abbreviated names for the genes are defined in Figure 3. Each data point represents the mean \pm SD of three replicates.

plays a key role in converting $18: 1$ to $18: 2$ to meet the demand of maintaining a basic level of 18:2 in lesquerella pollen. Similar results was reported for a safflower FAD2-10 that was expressed at a higher level in flower than cotyledon, hypocotyl, root, leaf and seed [70]. In addition, a FAD2 from Brassic napus was also found to be highly expressed in flower buds, and the expression was associated with membrane lipids and storage oil biosynthesis in pollen [71]. Recently, PfFAD2 has been shown to encode functional $\Delta 12$ desaturase activity in transformed yeast [69]. Although the bi-functional enzyme encoded by the PfFAH12 gene also has some oleate 12 -desaturase activity $[43,45]$ that could convert some of the $18: 1$ to $18: 2$, we suggest that PfFAD2 plays an essential role in the desaturation of $18: 1$ in all cells and tissues.

Interestingly FAD3 transcripts were detected with the highest number (59 isotigs) among all genes involved in fatty acid and TAG biosynthesis (Table 5). These FAD3 may catalyze PC-18:2 to PC-18:3 and/or PC-18:1-OH to PC-18:2-OH in lesquerella (Figure 3). Result of amino acid alignment of the isotigs indicated that there are two FAD3 isoforms in lesquerella compared to single FAD3 gene in Arabidopsis (Figure 5A, 5B). PfFAD3-1, represented by isotig17127, had 93-95\% identity to Arabidopsis AtFAD3 and Brassica napus BnFAD3a, BnFAD3b, and BnFAD3c [71], indicating a common FAD3 in Brassicaceae plants. However, PfFAD3-2, represented by isotig00061, is an isoform diverged from all the above $F A D 3$ s, with only $78-81 \%$ identity to AtFAD3 and BnFDA3s (Figure 5B). Isotig17127 and isotig00061 showed distinct $\mathrm{N}$-terminal coding regions (Figure 5A). Our expression profiling studies indicate that the expression of PfFAD3-1 progressively increased during seed development reaching a peak at 35 DAP, and then dropped sharply to undetectable levels at late stages 42 and 49 DAP (Figure 5C). The induction between 14 DAP and 35 DAP was 33-fold. Moderate levels of expression were detected in leaf, stem and root. In flower bud, the expression was at about 6-fold higher than in seeds at 14 DAP (Figure 5C). The temporal and spatial expression pattern of PfFAD3-2 is quite different from that of PfFAD3-1 (Figure 5D). At early stages of seed development (14-21 DAP), the expression of PfFAD3-2 was high and then declined quickly when seeds progressed to mid- and late-stages. No expression was detected in leaf, stem, root and flower buds. In our seed transcriptome (30 DAP), many more PfFAD3-2 transcripts were detected (58 isotigs) than LfFAD3-1 (1 isotig) (Table 5). This could be due to that the sample used for transcriptome study represented relatively young seeds. Based on the expression profiles of these two PfFAD3 genes, we suggest that both of them may contribute to the 
A AtFAD3 MVVAMDQRT NVINGDPG-AGD RKEERFDPSAQPPFKI GDI RAAI PKHCWKSPLRSMSYV 59
PFFAD3-1 MVVAMDKRSNVKGDPGGAGD KKEERFDPSAQPPFKI GDI RAAI PKHCWWKSPLRSMSY 60

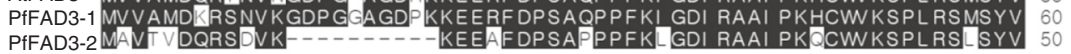

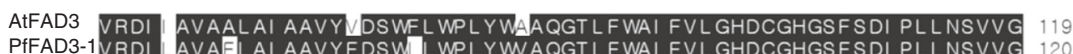

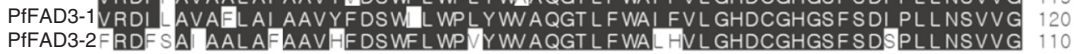
AtFAD3 HI LHSFI LVPYHGWRI SHRTHHQNHGHVENDESW PLPERVYKLLPHSTRMLRYTVPLP- 178 $\begin{array}{ll}\text { PFFAD3-1 HI LHSFI LVPYHGWRI SHRTHHQNHGHVENDESW PLPEKVY KNLPHST RML RYTVPLP- } & 179 \\ \text { PFFAD3-2HI LHSFI LVPFHGWRI SHKLHHQNHGHVENDESWP SEKVYKNMSRTTRMLRYTIII FS } 170\end{array}$ AtFAD3 MLAYPLYLCYRSPGKEGSHFNPYSSLFAPSERKLI ATSTTCWSI MFVILI ALSFVFGPLA 238

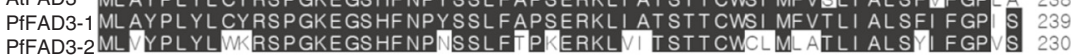
AtFAD3 VLKVYGVPYI I FVMM DAVTYLHHHGHDEKL PWYRGKEWSYLRGGLTTI DRDYGI FNNI H 298 PFFAD3-1 VL KVY GVPY I I FVMM DAVTYL HHHGHDEKL PWW RGKEWS L RGGL TTI DRDYGI FNNI H 299 ATFAD3 HDI GTHVI HHLFPOI PHYHLVDATKAAKHVL GRYYREPKTSGAI PI HLVESLVASI KKDH 358 PAFAD3-1 HDI GTHVI HHLFPQI PHYHLVEATKAAKHVLGYYREPKTSGAI PI HLVESLVSSI KKDH 350 PFFAD-2 HYCGTHVI HHLFPQI PHYHLVEAT NAAKHVIMGKYREPK KS GAI PVHLVESLFSSIKKNH 350 ATFAD3 YVSDTGDI VFYETDPOLYVYASDKSKI

$\begin{array}{ll}\text { PFFAD3-1 YVSDSGDI VFYETDPDLYVYASDKSKIN } & 387 \\ \text { PFFAD3-2YVSDTGDI VFYKTDPDLQDAYDKTKVN } & 378\end{array}$
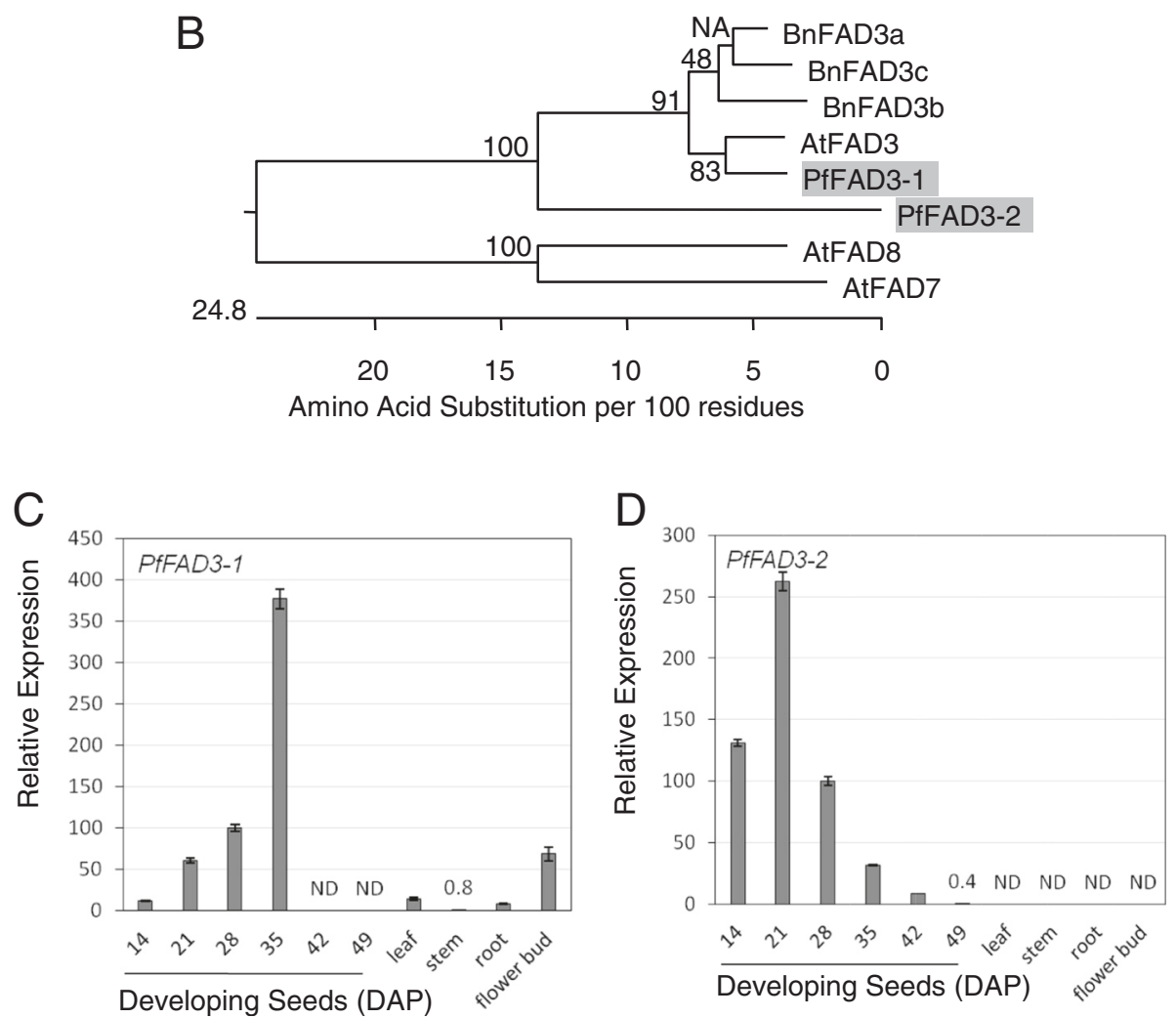

Figure 5 Characterization of two PfFAD3. (A) Amino acid sequences alignment among Arabidopsis FAD3 (AtFAD3:At2g29980), PfFAD3-1 (isotig17127) and PfFAD3-2 (isotig00061). Black shading indicates identical amino acids. No shading indicates dissimilar amino acids. Dashes indicate gaps in alignment. (B) Phylogenetic tree showing relationships among FAD3 and FAD7/8 desaturases. Lesquerella sequences are shaded. This tree was constructed by DNASTAR MegAlign program using the ClustalW method. Bootstrap used trials $=1000$, seed $=111$. At, Arabidopsis, Bn, Brassica napus FAD3a (AFJ19040), FAD3b (AFJ19037) and FAD3C (AFJ19034). Arabidopsis FAD7 (At3g11170) and FAD8 (At5g05580) used for outgroup. Expression of PfFAD3-1 (C) and PfFAD3-2 (D) in developing seeds and major organs determined by qPCR. Abbreviated names for the genes are described in Figure 3. Each data point represents the mean \pm SD of three replicates. ND = not detected. 
desaturation of FA in developing seeds, but PfFAD3-1 had a pattern similar to that of Arabidopsis (microarray data), and thus it may have a more general role in converting 18:2 to 18:3 in other organs. Since lesquerella seeds accumulate moderate amounts of $18: 2-\mathrm{OH}$ and $20: 2-\mathrm{OH}$, the divergent and also seed-specific PfFAD3-2 isoform might have evolved to desaturate 18:1-OH and 20:1$\mathrm{OH}$. Further functional characterization of PfFAD3-1 and PfFAD3-2 needs to be carried out in order to determine their enzymatic activity and substrate specificity in acyl-lipid metabolism.

\section{Conventional Kennedy pathway for TAG synthesis in ER}

The conventional Kennedy pathway for TAG synthesis utilizes three acyl-CoA-dependent acyltransferases, GPAT, LPAT and DGAT, that sequentially acylate the $s n$ 1 - and $s n-2$ - and then $s n$-3-position of G3P with acylCoA (Figure 3, red arrows). Since the synthesis of membrane glycerolipids also begins with sequential acylation of the $s n-1$ - and $s n-2$ - positions of G3P, GPAT and LPAT are common to synthesis of TAG and membrane glycerolipids. Using a bioinformatics approach, a new GPAT (At5g60620) was identified in Arabidopsis that exhibited extensive homology with a GPAT from mammalian cells involved in storage oil formation; that GPAT was postulated to be a putative AtGPAT9 for ER associated membrane and storage lipid biosynthesis in plants [72]. For the second acyl-CoA transferase, Arabidopsis LPAT2 (At3g57650) was found to be an ER-localized and involved in TAG and membrane lipid biosynthesis [73]. Using Arabidopsis genes (At5g60620 and At3g57650), we identified lesquerella orthologs of PfGPAT9 and PfLPAT2, each represented by 2 isotigs (Table 5). Result of gene expression analysis indicated that both genes were expressed at a similar level in most samples examined, including leaf, stem, flower bud, and developing seeds from 14 DAP to 42 DAP, with the exception of PfLPAT2 levels in stem tissue, where expression was only about $50 \%$ that detected in leaf (Figure 4B, 4C). Low levels of expression were detected in root and developing seeds at 49 DAP (Figure 4B, 4C). Our spatial and temporal expression profiles of PfGPAT9 and PfLPAT2 were similar to those from Arabidopsis [59,73-75]. Based on the overall spatial and temporal expression profiles of PfGPAT9 and PFLPAT2, we suggest both genes playing essential housekeeping roles in membrane and storage lipid biosynthesis throughout plant life.

DGAT catalyzes the final and rate-limiting step of TAG biosynthesis (Figure 3). There are three sequenceunrelated classes of DGATs reported in plants: membrane bound DGAT1 and DGAT2, and cytosolic DGAT3 (review) [76]. Using Arabidopsis DGAT1 (At2g19450), DGAT2 (At3g51520), and DGAT3 (At1g48300) as queries, seven lesquerella orthologs were identified in the seed transcriptome (Table 5). Phylogenic analysis among $D G A T$ genes from lesquerella, Arabidopsis, castor bean and peanut shows that the membrane type 1 (DGAT1), type 2 (DGAT2) and type 3 (DGAT3) are divided into three different clades (Figure 6A). The result of amino acid alignment of the isotigs indicates that there are two PfDGAT1 isoforms in lesquerella compared to single DGAT1 gene in Arabidopsis (Figure 6A, Additional file 1: Figure S1). Isotig11156 and isotig11157 had overall similarities in nucleotide and amino acid (AA) sequences (Table 5), but the first $100 \mathrm{AA}$ at the $\mathrm{N}$-termini show $45 \%$ divergence for isotig 11156 and $53 \%$ for isotig 11157 (Additional file 1: Figure S1). We designate the isoform encoded by isotig11157 as PfDGAT1-1 and that encoded by isotig11156 as PfDGAT1-2. Lesquerella DGAT2 is more related to the DGAT2 from Arabidopsis than to that from castor (Figure 6A). Protein sequence alignment revealed that castor DGAT2 has additional 25 AA at its N-terminus (Additional file 1: Figure S2). Soluble DGAT3 is divided into a distinct clade in the phylogeny tree from DGAT1 and DGAT2 due to sequence distance (Figure 6A). PfDGAT3 encodes a longer protein (360 AA) compared with those of 285, 332, and 345 AA of Arabidopsis, castor bean, and peanut DGAT3, respectively (Additional file 1: Figure S3). Protein sequence analysis showed that PfDGAT3 had $82 \%$ identity with AtDGAT3, even though it has 75 more AA (Additional file 1: Figure S3).

It is general accepted that depending on the plant species, DGAT1 or DGAT2 is a major enzyme responsible for the accumulation of seed TAG [76]. DGAT3 was recently demonstrated to be active in recycling of $18: 2$ and 18:3 FAs into TAG through a cytosolic pathway in peanut [77]. Our results of gene expression analysis showed that PfDGAT1-1 and PfDGAT1-2 had distinct expression patterns. PfDGAT1-1 was expressed in all stages during seed development and in leaf, stem, and flower bud, but it was expressed more in leaf, stem, and in immature seeds prior to active oil biosynthesis and became a predominant DGAT mRNA at late-maturation/desiccation stages (42-49 DAP) (Figure 6B). In contrast, PfDGAT1-2 had expression levels higher in developing seeds than in other tissues such as leaf, stem, root and flower buds (Figure 6C). PfDGAT1-2 may specifically contribute to TAG synthesis in seed. Indeed, our PfDGAT1-2 is the same gene as PfDGAT1a identified in a lesquerella seed cDNA library [78] and found to complement the Arabidopsis AS11 mutant [79]. AS11 had reduced DGAT activity and seed oil content due to a deletion in AtDGAT1 gene [79-81]. Seed-specific over-expression of an Arabidopsis cDNA encoding AtDGAT1 not only restored the oil content in AS11 but also enhanced seed oil content and seed weight in wild-type plants [82]. The expression profile of PfDGAT2 was overall similar to that of 


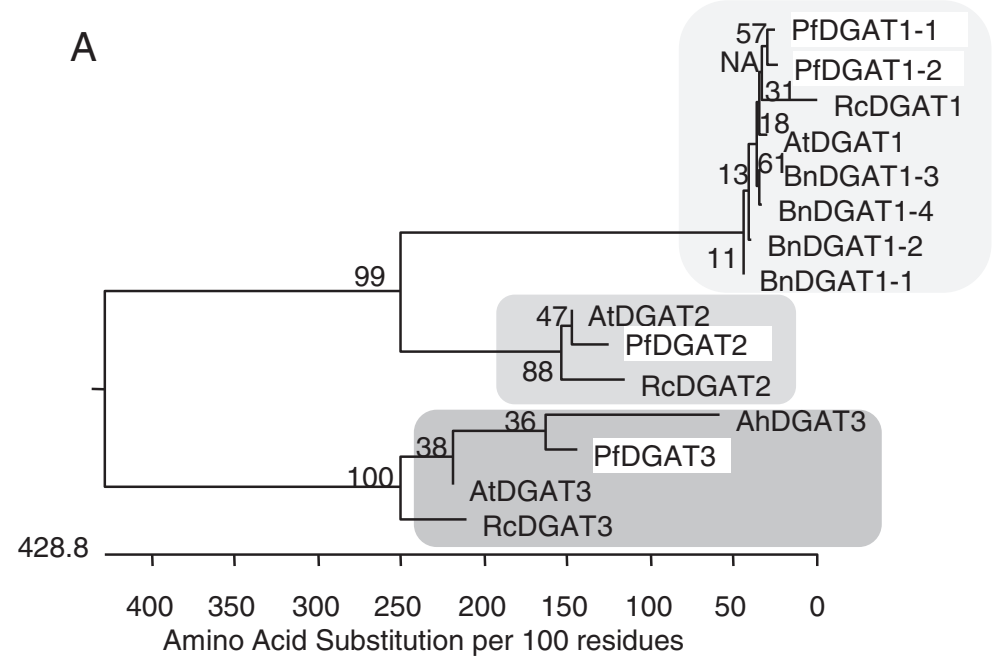

B
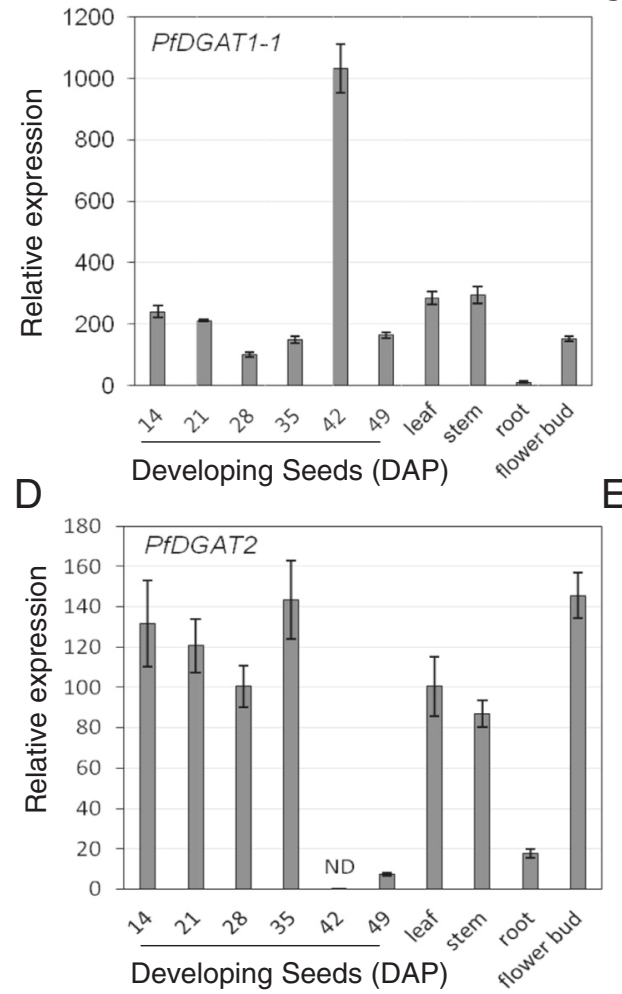

C

$\mathrm{E}$
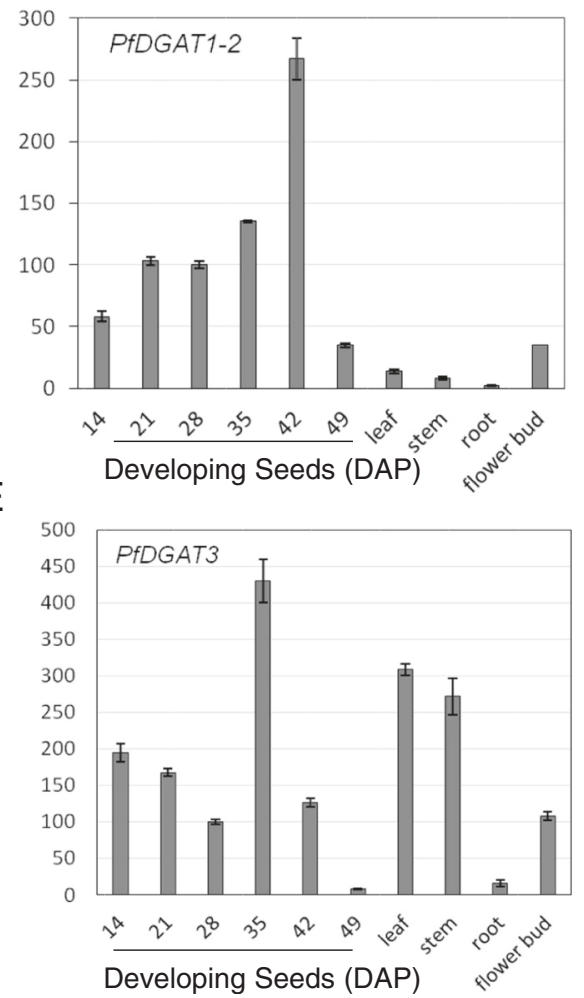

Figure 6 Characterization of three types PfDGATs. (A) Phylogenetic tree showing relationships among DGAT1, DGAT2, and DGAT3 from Physaria fendleri (Pf), Arabidopsis (At), Brassica napus (Bn), castor bean (Rc), peanut (Ah). PfDGAT1-1 (isotig11157), PfDGAT1-2 (isotig11156), PfDGAT2 (isotig19956), PfDGAT3 (isotig08903), AtDGAT1 (At2g19450), AtDGAT2 (At3g51520), AtDGAT3 (At1g48300), BnDGAT1-1 (AlA77019), BnDGAT1-2 (AIA67020), BnDGAT1-3 (AFM31260), BnDGAT1-4 (AAF64065), RcDGAT1 (XP_002514132), RcDGAT2 (XP_002528531), RcDGAT3 (XP_002519339), AhDGAT3 (AAX62735). The tree was constructed as described in Figure 5B. (B-E) Expression of PfDGAT1-1, PfDGAT1-2, PfDGAT2, and PfDGAT3 in developing seeds and major organs determined by qPCR. Abbreviated names for the genes are described in Figure 3. Each data point represents the mean \pm SD of three replicates. 
PfDGAT1-1, except in the late-maturation/desiccation stages where PfDGAT2 expression dropped to undetectable levels or trace amounts ( 42 and 49 DAP, respectively) (Figure 6D). The results indicate that both PfDGAT1-1 and PfDGAT2 may function in other physiological processes besides seed oil synthesis, and that they clearly contribute differently in lipid metabolism during late-maturation/desiccation stages of seed development. PfDGAT3 was ubiquitously expressed in all samples and showed a moderate dynamic pattern compared with the other PfDGATs. In leaf, stem, flower bud, and developing seeds at early stages (14-21 DAP), PfDGAT3 transcripts were detected at levels similar to that of PfDGAT1-1 (Figure 6B, 6E). In developing seeds at 35 DAP, their levels rose 2- to 4-fold before declining steadily at late stages 42-49 DAP (Figure 6B, 6E). The boosted expression of PfDGAT3 may be associated with increasing demands of membrane and storage lipids synthesis at 35 DAP, when seeds had attained their maximum size and storage compounds have accumulated to a high plateau [52]. The temporal and spatial expression pattern of PfDGAT3 suggests its role of house-keeping in most organs of lesquerella. Similar expression profile was reported for DGAT3 in peanut [83], Arbidopsis [75] and tung tree [84]. None of these DGAT3s were hypothesized to play a significant role in seed oil synthesis; rather it was proposed that they are involved in general TAG metabolism. Among all samples, root tissue had the lowest number of transcripts of all PfDGATs. While it is clear that PfDGAT1-2 plays a role in seed TAG assembly, it remains an open question as to whether or not PfDGAT1-1, PfDGAT2 or PfDGAT3 also contribute. Measurements of enzyme activity and substrate specificity in various tissues are needed to better elucidate the functions of the different PfDGATs. The results of such studies combined with our sequence characterization and expression profiling will provide the molecular basis for future identification of PfDGAT candidates for genetic engineering oilseeds for hydroxy fatty acid production.

\section{PC-mediated TAG synthesis}

As described, PC is the substrate for many FAmodifying enzymes (desaturase, hydroxylase, etc.). The FA fluxes into and out of $\mathrm{PC}$ are crucial for the production of TAG esterified with modified FAs, such as HFA. Based on current knowledge, there are three routes allowing PC-derived FA to be incorporated into TAG. First, The FA esterified to PC undergoes constant deacylation and reacylation by LPCAT in so called acyl editing [27]. Thus modified FA released by LPCAT can enter Kennedy pathway for TAG assembly (Figure 3, orange arrows). Second, direct transfer by PDAT of a FA from the $s n-2$ position of PC to the $s n-3$ position of DAG produces TAG (Figure 3, green arrows). Third, PDCT catalyzes the inter-conversion between DAG and PC by phosphocholine head group exchange (Figure 3, blue arrows). Thus FA on PC can be incorporated into the $s n-1$ and $s n-2$ positions of TAG by the PC derived DAG.

Recent studies have revealed the roles of the enzymes encoded by Arabidopsis LPCAT1 (At1g12640) and LPCAT2 (At1g63050) and other plant LPCATs genes [85-88]. They suggest that LPCATs are responsible for incorporation of newly synthesized fatty acid into PC (forward reaction), and transferring polyunsaturated and HFAs produced on PC directly to the acyl-CoA pool (reverse reaction) for further metabolism or catabolism. When reverse reactions were measured, a ricinoleoyl group at the $s n-2$ position of PC was removed 3- to 6fold faster than an oleoyl group by seven LPCATs from five species tested, including a PfLPCAT2 [88]. These results suggest an important role for PfLPCATs in removing 18:1-OH from $s n-2$ PC to an acyl-CoA pool. The mechanism of PC-modified UFAs channeled into TAG through efficient acyl editing and Kennedy pathway has been proposed as one of the major mechanisms that allow efficient assembling of UFA into TAGs [32]. As lesquerella TAGs contains $55-60 \% 20: 1-\mathrm{OH}$, it is likely that the acyl editing by PfLPCATs allows $18: 1-\mathrm{OH}$ to be immediately released from $\mathrm{PC}$, activated to $18: 1-\mathrm{OH}-$ CoA then elongated to 20:1-OH-CoA for further incorporation into TAG through the Kennedy pathway. When temporal and spatial expression patterns of Arabidopsis LPCATS were investigated using promoter fragments of AtLPCATs fused with the uidA reporter gene encoding $ß$-glucuronidase (GUS), differential expression patterns were observed for AtLPCAT1 and AtLPCAT2 [87]. Histochemical localization of GUS activity in transgenic lines showed that both promoters were active in seedling and rosette leaves. During seed development, AtLPCAT1 promoter activity was detected at a later developmental stage, whereas AtLPCAT2 promoter was active throughout seed development; it was also particularly strong in pollen grains. We have identified ten isotigs of PfLPCAT1 and one isotig of PfLPCAT2 in the lesquerella seed transcriptome, and found high sequence identity between lesquerella and Arabidopsis LPCATs (Table 5, Figure 7A). Our expression profiling data indicate that PfLPACTs are also differentially expressed (Figure 7B, 7C). During seed development, PfLPCAT1 showed a linear-rise throughout most stages up to 42 DAP, and then a sharp drop at 49 DAP when seeds enter the desiccation stage. The PfLPCAT1 temporal pattern resembles AtLPCAT1. Little expression of PfLPCAT1 was detected in leaf, stem, root and flower bud (Figure 7B). Unlike PfLPCAT1, PfLPCAT2 showed high expression during early (14-21) DAP stages of seed development, medium-high levels from 28-35 DAP, and then suddenly 

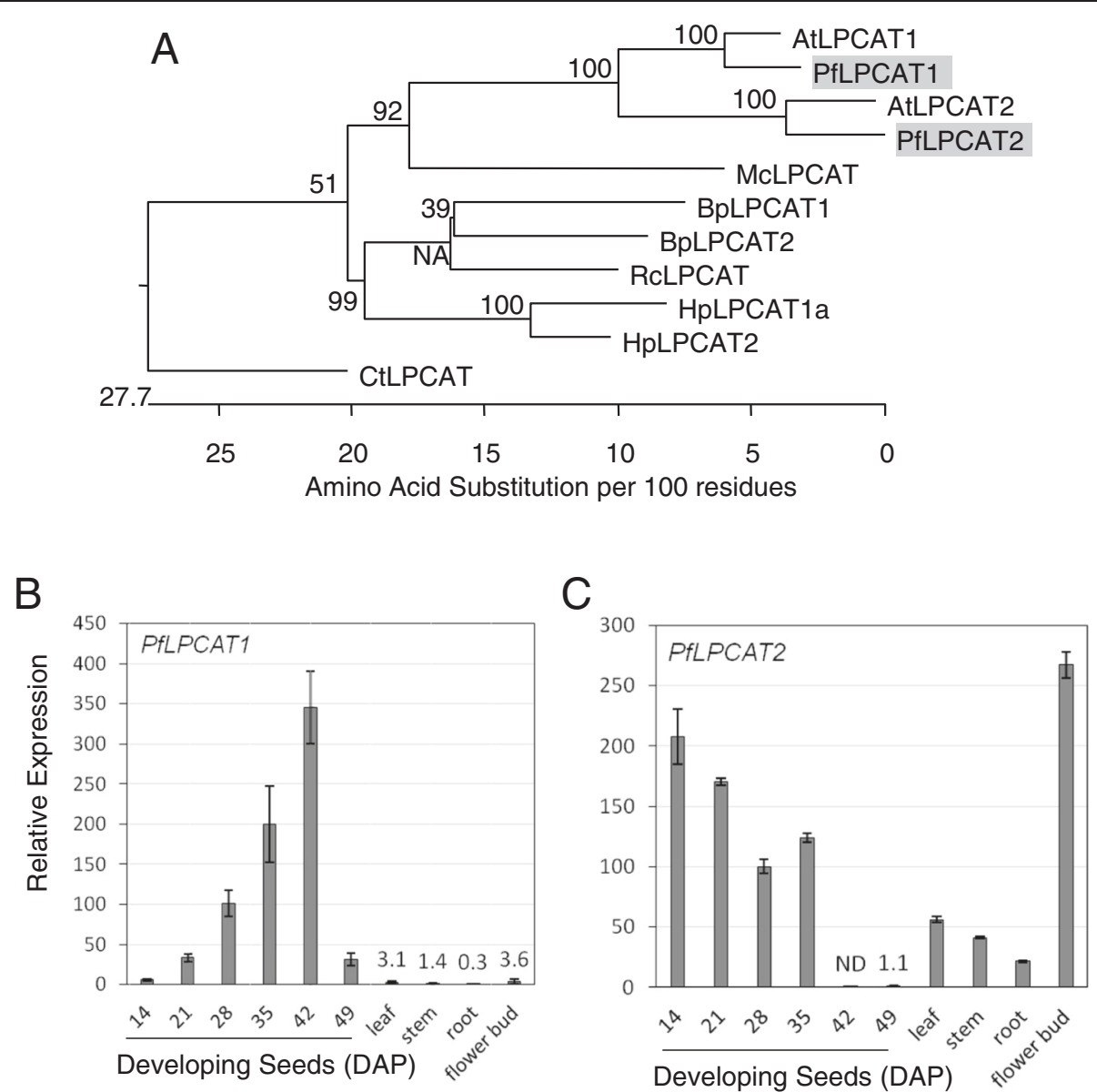

Figure 7 Characterization of two PfLPCATs. (A) Phylogenetic tree showing relationships among LPCAT1 and LPCAT2 from Physaria fendleri (Pf), Arabidopsis (At), Bernardia pulchella (Bp), castor bean (Rc), Hiptage benghalensis (Hb), Momordica charantia (Mc), Carthamus tinctorius (Ct). PfLPCAT1 (isotig03769), PfLPCAT2 (isotig16468), BpLPCAT1 (AHE80984), BpLPCAT2 (AHE80985), RCLPCAT (AHF20951), HpLPCAT1a (AHE80986), HpLPCAT2 (AHE80988), MCLPCAT (AGL81301), CtLPCAT (AHE80989). The tree was constructed as described in Figure 5B. (B, C) Expression of PfLPCAT1 and PFLPCAT2 in developing seeds and major organs determined by GPCR. Abbreviated names for the genes are described in Figure 3. Each data point represents the mean \pm SD of three replicates.

became undetectable and extremely low at late stages 42 and 49 DAP, respectively (Figure 7C). In leaf, stem and root, moderate expression of PfLPCAT2 was detected (Figure 7C). In flower bud, PfLPCAT2 was expressed highest among all organs investigated. (Figure $7 \mathrm{C}$ ). The overall expression profile of PfLPCAT2 is similar to that of AtLPCAT2 based on promoter activity analysis [83] and information available at the Arabidopsis eFP browser [75]. Our results revealed that both PfLPCATs were expressed during seed development but with opposite trends. Thus, it is likely that both PfLPCATs contribute to TAG metabolism in lesquerella seeds. PfLPCAT2 was highly expressed in flower bud, suggesting its involvement in TAG synthesis in pollen.

PDAT was identified and characterized as an acylCoA-independent transacylase that transfers the fatty acid at the $s n-2$ position in PC to the $s n-3$ position of DAG, thus synthesizing TAG [37]. Two PDATs were found from Arabidopsis, PDAT1 (At5g13640) and PDAT2 (At3g44830) [89]. AtPDAT1 is ubiquitously expressed [75,90], but AtPDAT2 transcripts are predominantly found in seed [75]. Together with AtDGAT1, AtPDAT1 has been shown to determine the amount of TAG synthesis in pollen grains and developing seeds, whereas AtPDAT2 does not play a substantial role in TAG synthesis, although it is highly expressed during seed development [91]. Based on homology searches with Arabidopsis PDATs, we identified a total of 28 isotigs of PfPDAT transcripts in the lesquerella seed transcriptome. Detailed comparison among 28 isotigs led to discovery of PfPDAT1-1, PfPDAT1-2, and PfPDAT2 (Table 5). PfPDAT1-2 is slightly closer to AtPDAT1 than PfPDAT1-1 (Table 5, Figure $8 \mathrm{~A}$ ). Sequence alignments revealed that the 3'-UTR's of PfPDAT1-1 and PfPDAT1-2 were distinct (Additional file 1: Figure S4). Gene expression analysis revealed that PfPDAT1-1 was ubiquitously expressed in 

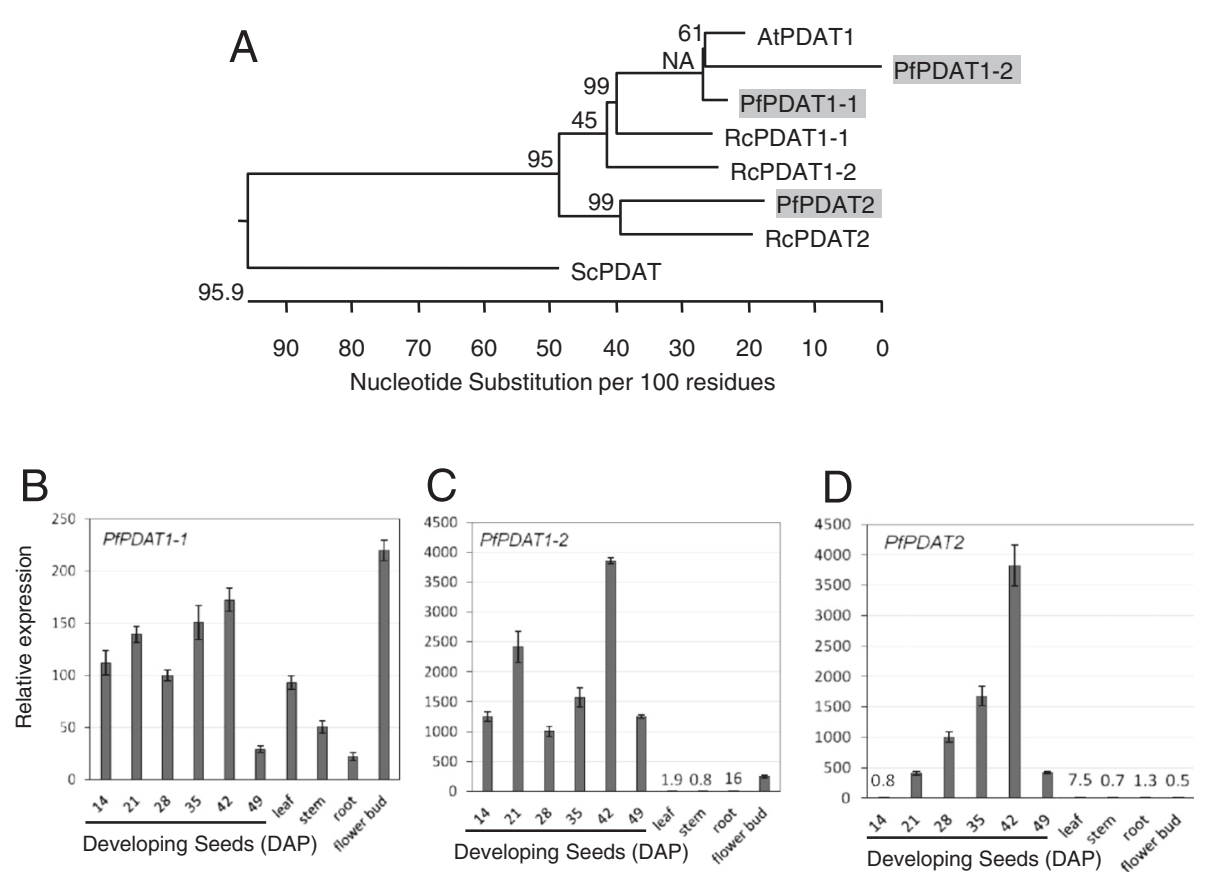

Figure 8 Characterization of three PfPDATs. (A) Phylogenetic tree of PDATs from Physaria fendleri (Pf), Arabidopsis (At), castor bean (Rc), Saccharomyces cerevisiae (Sc). PfPDAT1-1, PfPDAT1-2, PfPDAT2, AtPDAT1 (At5g13640), AtPDAT2(At3g44830), RcPDAT1-1 (HM807520), RcPDAT1-2 (HM807521), RCPDAT2 (HM807522), SCPDAT (NM_001183185). The tree was constructed as described in Figure 5B. (B-D) Expression of PfPDAT1-1, PFPDAT1-2, and PFPDAT2 in developing seeds and major organs determined by qPCR.Abbreviated names for the genes are described in Figure 3. Each data point represents the mean \pm SD of three replicates.

developing seeds at all stages and in other organs examined (Figure $8 \mathrm{~B}$ ), similar to the expression pattern of AtPDAT1. Expression levels of PfPDAT1-2 were about 100 -fold higher in developing seeds compared with PfPDAT1-1, but were negligible in leaf, stem and root (Figure 8C). In flower bud, both PfPDAT1-1 and PfPDAT1-2 isoforms were highly expressed at equivalent (Figure 8B, 8C). The temporal expression of PfPDAT2 showed an linear rise during seed development up to 42 DAP, and then a drop to a low level at the latest stage 49 DAP. The highest level at $42 \mathrm{DAP}$ is comparable to that of PfPDAT1-2. In leaf, stem, root and flower bud, the expression level of PfPDAT2 was negligible (Figure 8D). The role of PDAT in HFAs synthesis has been well studied in castor by two groups [50,51], who both found that castor PDATs were comprised of three members, RcPDAT1-1 [51] (same as RcPDAT1B [50]), RcPDAT1-2 [51] (same as RcPDAT1A [50]) and RcPDAT2. Gene expression analysis indicated that RcPDAT1-1/RcPDAT1B and RcPDAT2 had profiles similar to those of AtPDAT1 and AtPDAT2, respectively, [50,51]. However, RcPDAT1-2/RcPDAT1A was highly expressed in developing castor seeds, and this isoform was not found in Arabidopsis. Since castor accumulates 90\% HFA in seed TAG, it has been proposed that the enzyme encoded by RCPDAT1-2/RcPDAT1A could be important for HFA-TAG synthesis. Indeed both groups demonstrated that RcPDAT1-2/RcPDAT1A facilitated the transfer of HFAs from PC into TAG in transgenic Arabidopsis. Unlike castor oil which has over $70 \%$ of TAGs esterified with 18:1-OH in all three positions [92], lesquerella TAGs contain $55-60 \% 20: 1-\mathrm{OH}$, and majority of them are esterified in $s n-1$ and $s n-3$ positions [3]. The lack of HFA at the $s n-2$ of TAG could be explained by LPATs in lesquerella discriminating 20:1-OH substrate. Recent studies of transgenic Camelina expressing RcFAH12 and PfKCS3 suggested the mechanism of 20:1-OH in TAG assembly mainly utilizing Kennedy pathway in lesquerella [93]. Therefore, PfPDATs are unlikely major enzymes channeling HFA into TAGs. The roles of PfPDATs remain to be determined. The gene sequence and expression profile of PfPDATs identified in this study provide critical information for future investigation on the enzymatic activity and substrate specificity in acyl-lipid metabolism in lesquerella.

Fatty acids at the $s n-1$ and $s n-2$ positions in $\mathrm{PC}$ can be directly transferred to TAG through DAG converted by PDCT. The PDCT enzyme, encoded in Arabidopsis by the Reduced Oleate Desaturation1 (ROD1) gene, catalyzes the inter-conversion between DAG and PC by phosphocholine head group exchange [36]. In castor, the $18: 1-\mathrm{OH}$ is produced by the hydroxylation of $18: 1$ that is esterified to the $s n-2$ position of PC [39]. Since PDCT catalyzes the shuffling of acyl groups between PC and DAG, it provides a mechanism of making HFA-DAG from HFA-PC, thus the HFA-DAG can be subsequently 
converted to HFA-TAG. A castor PDCT enzyme gene was isolated and co-expressed in a transgenic Arabidopsis line carrying RcFAH12. The doubly transformed line had increases of $17-23 \%$ in seed HFA content [35]. The authors noted that co-expression of AtPDCT did not increase HFA in transgenic Arabidopsis, indicating that RcPDCT had evolved to effectively convert HFA-PC to HFA-DAG [35]. In the lesquerella seed transcriptome, we have identified one isotig25038 showing high homology with Arabidopsis PDCT and have designated it PfPDCT. The PfPDCT sequence shares $89 \%$ and $73 \%$ identify with AtPDCT and RcPDCT, respectively (Additional file 1: Figure S5). Gene expression analysis indicated that PfPDCT is expressed ubiquitously in developing seeds and other organs examined (Figure 4D). Among most samples, the expression levels ranged from 100-243 relative copy number, with exception of the seed sample at 42 DAP and the root sample, which had levels of about 365 and 11, respectively. A similar expression profile for AtPDCT was reported [36]. It is known that the $s n-2$ position of TAG in lesquerella consists almost all of $\mathrm{C} 18$ unsaturated acyl groups including 18:1, 18:2 and 18:3 [3]. Thus PfPDCT would not be a major enzyme involved in channeling HFA into lesquerella TAGs. It is possible that PfPDCT contributes FA flux through PC-derived DAG in TAG assembly in lesquerella. However, based on the expression profile of PfPDCT, it is likely that PfPDCT plays a general housekeeping function in lesquerella acyl-lipid metabolism.

\section{Conclusions}

Lesquerella is valued for its unusual HFA in seeds. Deep sequencing of cDNAs from developing lesquerella seeds was carried out to identify candidate genes that are associated with the synthesis of seed TAG enriched with HFA. A total of 26,995 unique genes from 651 megabase raw sequences were assembled and $74 \%$ of them $(19,861)$ had homology with known genes. The vast majority $(95 \%, 18,868)$ of the matched genes showed highest homology to Arabidopsis genes, confirming the close relationship between the two species. The results provide a molecular basis for translating findings from the model plant Arabidopsis to facilitate lesquerella crop improvement. Genes involved in the synthesis of FA and TAG were identified and annotated by detailed sequence alignments. We have identified nearly all of the known genes for de novo FA biosynthesis and export from the plastid, and all of the known genes for TAG assembly in ER. In addition, we characterized the temporal and spatial expression profiles of 15 key genes in TAG metabolism using quantitative RT-PCR. The sequence and gene expression data presented in this study will serve as a useful resource for future research on lesquerella and other oilseed crops and promote their development into safe sources of HFA.

\section{Methods}

Plant material and general growth conditions

The $P$. fendleri seeds, WCL-LY2 [4], were kindly provided by Dr. David Dierig (USDA-ARS, National Center for Genetic Resources Preservation, Fort Collins, Colorado 80521, USA). Plants were germinated and grown in a greenhouse at temperatures between $28^{\circ} \mathrm{C}$ (day) and $18^{\circ} \mathrm{C}$ (night), with supplemental metal halide lighting to provide a 15-h-day length (1000 to $1250 \mu \mathrm{mol} \mathrm{m}^{-2} \mathrm{~s}^{-1}$ ). Mature flowers were individually hand-pollinated and tagged, and the tagging dates were recorded as 0 day after pollination (0 DAP). Developing seeds at 7, 14, 21, $28,30,35,42$ and 49 DAP were frozen immediately in liquid nitrogen after harvest and stored at $-80^{\circ} \mathrm{C}$. Leaf and stem tissues were obtained from mature plants, and root tissue was obtained from 2 month old seedlings cultured in half-strength MS liquid medium [94]. Our flower sample consists of mature flower buds. Once the tissues were harvested, they were frozen immediately in liquid nitrogen and stored at $-80^{\circ} \mathrm{C}$.

\section{RNA preparation, CDNA library construction and sequencing}

Total RNA was extracted from developing seeds using TRIzol Reagent (Invitrogen, Carlsbad, CA). RNA pellets were dissolved in RNAse-free water, quantified by NanoDrop ND-1000 spectrophotometer (NanoDrop Technologies, Inc., Wilmington, DE). RNA quality was checked by $2 \%$ agarose gel electrophoresis. Total RNA from the 30 DAP sample was used for preparing an mRNA sample and subsequently constructing of a cDNA library using Illumina ${ }^{-} \mathrm{TruSeq}^{\mathrm{Tm}}$ RNA Sample Preparation Kit (Illumina Inc., San Diego, CA ). In brief, the mRNA was purified using poly-T oligo attached to magnetic beads. Following purification, the mRNA was fragmented into small pieces using divalent cations under elevated temperature. The cleaved RNA fragments were copied into first strand $\mathrm{CDNA}$ using reverse transcriptase and random primers. This was followed by second strand cDNA synthesis using DNA Polymerase I and RNase $H$. These cDNA fragments then went through an end repair process, the addition of a single ' $\mathrm{A}$ ' base, and then ligation of the adapters. The products were then purified and enriched with PCR to create the final cDNA library. The cDNA library was sequenced on a GS FLX Titanium sequencing platform (Roche, Branford, CT).

\section{Assembly and gene annotation}

High quality sequence reads from seed libraries were assembled into isotigs and singletons using GS De Novo Assembler (v 2.6) software with the option for de novo 
transcriptome assembly. Clean singletons were processed to obtain high quality clean sequences, SeqClean was used to trim adapter sequences and Lucy (version $1.20 \mathrm{p}$ ) was used to remove low quality sequences and those $<100 \mathrm{bp}$. As a result, total 21,912 singletons were generated.

To annotate the detected genes, a BLASTx search against the NCBI non-redundant protein (NR) database (http://www.ncbi.nlm.nih.gov/refseq/) was performed with an E-value threshold of less than $10^{-3}$. NR annotation was used to obtain GO annotation of genes according to molecular function, biological process and cellular component ontologies (http://www.geneontology.org/).

\section{Quantitative RT-PCR}

Total RNA was reverse transcribed using the QuantiTect Reverse Transcription Kit (QIAGEN, Valencia, CA) according to manufacturer's directions. The resulted cDNA samples were used in PCR reactions. Standard PCR amplification reactions were carried out in a volume of $25 \mu \mathrm{L}$ containing $20 \mathrm{ng}$ of cDNA, $0.5 \mu \mathrm{M}$ each of forward and reverse primers and $1 \times$ SYBR Select Master Mix, CFX (Applied Biosystems) using a 7500 Fast Real-Time PCR system (Applied Biosystems) and standard default thermal cycling conditions [initial step, $95^{\circ} \mathrm{C}$ for $10 \mathrm{~min}$ for polymerase activation; 40 cylces of PCR, $95^{\circ} \mathrm{C}, 15 \mathrm{~s}$ for melting, $60^{\circ} \mathrm{C}, 1 \mathrm{~min}$ for annealing and extending; and dissociation step set by the system software]. Putative oligonucleotide primers were designed using Primer Express, version 3 software (Applied Biosystems). To ensure maximum specificity and efficiency during quantitative PCR, putative primer pairs were further tested for linearity of response by constructing standard curves on five or six serial 10 -fold dilutions. The templates used for the standard curve analysis were mixed cDNAs from developing seeds, leaf and flower samples with a starting concentration of $20 \mathrm{ng} / \mu \mathrm{L}$. For each primer set, standard curves were analyzed independently at least three times, and standard curves repeatedly showing correlation coefficients of 0.99 or higher and PCR efficiencies between 83 and $107 \%$ were accepted. PCR product specificity was confirmed by melting-curve analysis and by electrophoresis on $4 \%$ agarose gel to ensure that PCR reactions were free of primer dimers and non-specific amplicons. Information on primer pairs and their PCR efficiencies is listed in Additional file 2: Table S1. The method of Pfaffl [95] was applied to calculate comparative expression levels between samples. The P. fendleri $18 \mathrm{~S}$ gene was used as internal reference to normalize the relative amount of RNAs for all samples. For each selected gene, triplicate sets of PCR reaction samples including the $18 \mathrm{~S}$ controls, and duplicate negative controls (reaction samples without cDNA templates), were prepared and run in a 96-well plate. The average $C_{T}$ from 28 DAP measurements were calibrated as 100 or 1000 copy numbers, and the relative copy numbers of a gene were averaged over triplicates. The PCR experiments were repeated three times for each plate to ensure that similar results could be obtained.

\section{Availability of supporting data}

The sequence raw data from this study have been submitted to the NCBI Sequence Read Archive (SRA) http://www.ncbi.nlm.nih.gov/bioproject/260225) under the BioProject ID PRJNA260225.

\section{Additional files}

Additional file 1: Figure S1. Protein sequence alignment among PfDGAT1-1 (isotig11157), PfDGAT1-2 (isotig11156), AtDGAT1 (At2g19450), and RCDGAT1 (XP_002514132). Black shading indicates identical amino acids. Gray shading indicates similar amino acids and no shading indicates dissimilar amino acids. Dashes indicate gaps in alignment. Figure S2. Protein sequence alignment among PfDGAT2 (isotig19956), AtDGAT2 (At3g51520), and RcDGAT2 (XP_002528531). Figure S3. Protein sequence alignment among PfDGAT3 (isotig08903), AtDGAT3 (At1g48300), and AhDGAT3 (AAX62735). Figure S4. C-terminal coding region of nucleotide sequence alignment between PfPDAT1-1 (isotig08780) and PfPDAT1-2 (isotig08781). Locations of qRT-PCR primer sequences for each gene are indicated with red arrows. Figure S5. A. Phylogenetic tree showing relatedness among PfPDCT (isotig25038) (shaded), AtPDCT (At3g15820) RCPDCT (XP_002517643). B. Amino acid sequence alignments among the same three sequences. PfPDCT is partial cDNA sequence.

Additional file 2: Table S1. The lesquerella gene primers used in the qPCR analysis.

\section{Competing interests}

The authors declare that they have no competing interests.

\section{Authors' contributions}

HUK and GQC designed the research, performed experiments, and wrote the paper. Both authors read and approved the final manuscript.

\section{Acknowledgements}

This study was conducted with support from the National Academy of Agricultural Science (project no. PJ01007505) and International Cooperative Research Project Program (Project No. PJ00855602) of the National Academy of Agricultural Science and "Next-Generation BioGreen 21 Program (SSAC, Project No. PJ01108101)" of the Rural development Administration (RDA), Republic of Korea, from the US Department of Agriculture-Agricultural Research Service-Current Research Information System Project 5325-21410-020-00D, and from the USDA Trust Fund Cooperative Agreement with RDA (Agreement number: 5802129036 F). The authors wish to thank Ann Blechl and Mark Smith for critical reading of the manuscript, and Kumiko Johnson for assisting qPCR analysis. Thanks also go to Dr. David Dierig and Dr. Mark Cruz for providing the cover photo of leaquerella grown in a research field of US Department of Agriculture. USDA is an equal opportunity provider and employer. Mention of a specific product name by the United States Department of Agriculture does not constitute an endorsement and does not imply a recommendation over other suitable products.

Received: 5 October 2014 Accepted: 27 February 2015

Published online: 24 March 2015

\section{References}

1. Al-Shehbaz IA, O'Kane Jr SL. Lesquerella is united with Physaria (Brassicaceae). Novon. 2002;12(3):319-29.

2. Isbell TA, Mund MS, Evangelista RL, Dierig DA. Method for analysis of fatty acid distribution and oil content on a single Lesquerella fendleri seed. Ind Crops Prod. 2008;28(2):231-6. 
3. Hayes DG, Kleiman R. A detailed triglyceride analysis of Lesquerella fendleri oil: column chromatographic fractionation followed by supercritical fluid chromatography. J Am Oil Chem Soc. 1996;73(2):267-9.

4. Dierig DA, Tomasi PM, Dahlquist GH. Registration of WCL-LY2 high oil Lesquerella fendleri germplasm. Crop Sci. 2001;41:604-5.

5. Chen GQ, Lin JT, Lu C. Hydroxy fatty acid synthesis and lipid gene expression during seed development in Lesquerella fendleri. Ind Crops Prod. 2010;34(2):1286-92.

6. Hayes DG, Carlson KD, Kleiman R. The isolation of hydroxy acids from lesquerella oil lipolysate by a saponification/extraction technique. J Am Oil Chem Soc. 1996;73(9):1113-9.

7. Caupin HJ. Products from castor oil: past, present and future. New York, NY: Marcel Dekker; 1997.

8. Goodrum JW, Geller DP. Influence of fatty acid methyl esters from hydroxylated vegetable oils on diesel fuel lubricity. Bioresour Technol. 2005;96(7):851-5.

9. Moser BR, Cermak SC, Isbell TA. Evaluation of Castor and Lesquerella oil derivatives as additives in biodiesel and ultralow sulfur diesel fuels. Energy and Fuels. 2008:22(2):1349-52.

10. Carlson KD, Chaudhry A, Bagby MO. Analysis of oil and meal from Lesquerella fendleri seed. J Am Oil Chem Soc. 1990;67(7):438-42.

11. Wu Y, Hojilla-Evangelista MP. Lesquerella fendleri protein fractionation and characterization. J Am Oil Chem Soc. 2005:82(1):53-6.

12. Abbott TP, Victor Wu Y, Carlson KD, Slodki ME, Kleiman R. Isolation and preliminary characterization of Lesquerella fendleri gums from seed, presscake, and defatted meal. J Agric Food Chem. 1994;42(8):1678-85.

13. Harry-O'kuru RE, Carriere CJ, Wing RE. Rheology of modified Lesquerella gum. Ind Crops Prod. 1999;10(1):11-20.

14. Holser RA, Carriere CJ, Abbott TP. Rheological properties of lesquerella gum fractions recovered by aqueous extraction. Ind Crops Prod. 2000;12(1):63-9.

15. Wu YV, Abbott TP. Enrichment of gum content from Lesquerella fendleri seed coat by air classification. Ind Crops Prod. 1996;5(1):47-51

16. Dierig DA, Thompson AE, Nakayama FS. Lesquerella commercialization efforts in the United States. Ind Crops Prod. 1993;1:289-93.

17. Dierig DA, Tomasi PM, Salywon AM, Ray DT. Improvement in hydroxy fatty acid seed oil content and other traits from interspecific hybrids of three Lesquerella species: Lesquerella fendleri, L. pallida, and L. lindheimeri. Euphytica. 2004;139(3):199-206.

18. Dierig DA, Adam NR, Mackey BE, Dahlquist GH, Coffelt TA. Temperature and elevation effects on plant growth, development, and seed production of two Lesquerella species. Ind Crops Prod. 2006;24(1):17-25.

19. Dierig DA, Wang G, McCloskey WB, Thorp KR, Isbell TA, Ray DT, et al. Lesquerella: new crop development and commercialization in the U.S. Ind Crops Prod. 2011;34(2):1381-5.

20. Chen GQ. Effective reduction of chimeric tissue in transgenics for the stable genetic transformation of lesquerella fendleri. HortScience. 2011;46(1):86-90.

21. Jenderek MM, Dierig DA, Isbell TA. Fatty-acid profile of Lesquerella germplasm in the National Plant Germplasm System collection. Ind Crops Prod. 2009:29:154-64.

22. Dierig DA, Thompson AE, Rebman JP, Kleiman R, Phillips BS. Collection and evaluation of new Lesquerella and Physaria germplasm. Ind Crops Prod. 1996;5(1):53-63.

23. Salywon AM, Dierig DA, Rebman JP, De Rodriguez DJ. Evaluation of new Lesquerella and Physaria (Brassicaceae) oilseed germplasm. Am J Bot. 2005:92(1):53-62

24. Reed DW, Taylor DC, Covello PS. Metabolism of hydroxy fatty acids in developing seeds in the genera Lesquerella (Brassicaceae) and Linum (Linaceae). Plant Physiol. 1997;114(1):63-8.

25. Engeseth N, Stymne S. Desaturation of oxygenated fatty acids in Lesquerella and other oil seeds. Planta. 1996;198(2):238-45

26. Chapman KD, Ohlrogge JB. Compartmentation of triacylglycerol accumulation in plants. J Biol Chem. 2012:287(4):2288-94.

27. Bates PD, Stymne S, Ohlrogge J. Biochemical pathways in seed oil synthesis. Curr Opin Plant Biol. 2013:16(3):358-64.

28. Weiss SB, Kennedy EP. The enzymatic synthesis of triglycerides. J Am Chem Soc. 1956;78(14):3550.

29. Weiss SB, Kennedy EP, Kiyasu JY. The enzymatic synthesis of triglycerides. J Biol Chem. 1960;235:40-4

30. Lands WE. Lipid metabolism. Annu Rev Biochem. 1965;34:313-46.
31. Li-Beisson $Y$, Shorrosh B, Beisson F, Andersson MX, Arondel V, Bates PD, et al. Acyl-lipid metabolism. The Arabidopsis Book / American Society of Plant Biologists. 2013;11:e0161.

32. Bates PD, Browse J. The significance of different diacylgycerol synthesis pathways on plant oil composition and bioengineering. Front Plant Sci. 2012;3:147.

33. Stymne S, Stobart AK. Evidence for the reversibility of the acyl-CoA: lysophosphatidylcholine acyltransferase in microsomal preparations from developing safflower (Carthamus tinctorius L.) cotyledons and rat liver Biochemical J. 1984;223(2):305-14.

34. Ståhl U, Stålberg K, Stymne S, Ronne H. A family of eukaryotic lysophospholipid acyltransferases with broad specificity. FEBS Lett. 2008;582(2):305-9.

35. Hu Z, Ren Z, Lu C. The phosphatidylcholine diacylglycerol cholinephosphotransferase is required for efficient hydroxy fatty acid accumulation in transgenic Arabidopsis. Plant Physiol. 2012;158(4):1944-54.

36. Lu C, Xin Z, Ren Z, Miquel M, Browse J. An enzyme regulating triacylglycero composition is encoded by the ROD1 gene of Arabidopsis. Proc Natl Acad Sci U S A. 2009:106(44):18837-42.

37. Dahlqvist A, Stahl U, Lenman M, Banas A, Lee M, Sandager $L$, et al. Phospholipid:diacylglycerol acyltransferase: an enzyme that catalyzes the acyl-CoA-independent formation of triacylglycerol in yeast and plants. Proc Natl Acad Sci U S A. 2000:97(12):6487-92.

38. Lee K-R, Chen GQ, Kim HU. Current progress towards the metabolic engineering of plant seed oil for hydroxy fatty acids production. Plant Cell Rep. 2015. doi 10.1007/s00299-015-1736-6

39. Bafor M, Smith MA, Jonsson L, Stobart K, Stymne S. Ricinoleic acid biosynthesis and triacylglycerol assembly in microsomal preparations from developing castor-bean (Ricinus communis) endosperm. Biochemical J. 1991;280(2):507-14.

40. Moreau RA, Stumpf PK. Recent studies of the enzymic synthesis of ricinoleic Acid by developing castor beans. Plant Physiol. 1981;67(4):672-6.

41. Moon H, Smith MA, Kunst L. A condensing enzyme from the seeds of Lesquerella fendleri that specifically elongates hydroxy fatty acids. Plant Physiol. 2001;127(4):1635-43.

42. Van De Loo FJ, Broun P, Turner S, Somerville C C. An oleate 12-hydroxylase from Ricinus communis $L$. is a fatty acyl desaturase homolog. Proc Nat Acad Sci U S A. 1995:92(15):6743-7.

43. Broun P, Boddupalli S, Somerville C. A bifunctional oleate 12-hydroxylase: desaturase from Lesquerella fendleri. Plant J. 1998;13(2):201-10.

44. Smith MA, Moon H, Chowrira G, Kunst L. Heterologous expression of a fatty acid hydroxylase gene in developing seeds of Arabidopsis thaliana. Planta. 2003:217(3):507-16.

45. Broun P, Shanklin J, Whittle E, Somerville C. Catalytic plasticity of fatty acid modification enzymes underlying chemical diversity of plant lipids. Science. 1998;282(5392):1315-7.

46. Broun P. Somerville C. Accumulation of ricinoleic, lesquerolic, and densipolic acids in seeds of transgenic arabidopsis plants that express a fatty acy hydroxylase cDNA from castor bean. Plant Physiol. 1997;113(3):933-42.

47. Kumar R, Wallis JG, Skidmore C, Browse J. A mutation in Arabidopsis cytochrome b5 reductase identified by high-throughput screening differentially affects hydroxylation and desaturation. Plant J. 2006;48(6):920-32.

48. Lu C, Fulda M, Wallis JG, Browse J. A high-throughput screen for genes from castor that boost hydroxy fatty acid accumulation in seed oils of transgenic Arabidopsis. Plant J. 2006:45(5):847-56.

49. Burgal J, Shockey J, Lu C, Dyer J, Larson T, Graham I, et al. Metabolic engineering of hydroxy fatty acid production in plants: RcDGAT2 drives dramatic increases in ricinoleate levels in seed oil. Plant Biotechnol J. 2008;6(8):819-31.

50. van Erp H, Bates PD, Burgal J, Shockey J, Browse J. Castor phospholipid: diacylglycerol acyltransferase facilitates efficient metabolism of hydroxy fatty acids in transgenic Arabidopsis. Plant Physiol. 2011:155(2):683-93.

51. Kim HU, Lee KR, Go YS, Jung JH, Suh MC, Kim JB. Endoplasmic reticulum-located PDAT1-2 from castor bean enhances hydroxy fatty acid accumulation in transgenic plants. Plant Cell Physiol. 2011;52(6):983-93.

52. Chen GQ, Vang L, Lin JT. Seed development in Lesquerella fendleri (L.). HortScience. 2009;44(5):1415-8.

53. Yang P, Li X, Shipp MJ, Shockey JM, Cahoon EB. Mining the bitter melon (momordica charantia I) seed transcriptome by 454 analysis of non-normalized and normalized cDNA populations for conjugated fatty acid metabolism-related genes. BMC Plant Biol. 2010;10:250. 
54. Zhou Y, Gao F, Liu R, Feng J, Li H. De novo sequencing and analysis of root transcriptome using 454 pyrosequencing to discover putative genes associated with drought tolerance in Ammopiptanthus mongolicus. BMC Genomics. 2012;13:266.

55. Liang C, Liu X, Yiu SM, Lim BL. De novo assembly and characterization of Camelina sativa transcriptome by paired-end sequencing. BMC Genomics. 2013;14:146.

56. Nguyen HT, Silva JE, Podicheti R, Macrander J, Yang W, Nazarenus TJ, et al. Camelina seed transcriptome: a tool for meal and oil improvement and translational research. Plant Biotechnol J. 2013;11(6):759-69.

57. SILVA rRNA database. http://www.arb-silva.de/.

58. Arabidopsis acyl-lipid metabolism database. http://arabidopsisacyllipids. plantbiology.msu.edu/pathways/pathways.

59. Schmid M, Davison TS, Henz SR, Pape UJ, Demar M, Vingron M, et al. A gene expression map of Arabidopsis thaliana development. Nat Genet. 2005;37(5):501-6.

60. Li X, llarslan H, Brachova L, Qian HR, Li L, Che P, et al. Reverse-genetic analysis of the two biotin-containing subunit genes of the heteromeric acetyl-coenzyme A carboxylase in Arabidopsis indicates a unidirectional functional redundancy. Plant Physiol. 2011;155(1):293-314.

61. Hlousek-Radojcic A, Post-Beittenmiller D, Ohlrogge JB. Expression of constitutive and tissue-specific acyl carrier protein isoforms in Arabidopsis. Plant Physiol. 1992;98(1):206-14

62. Carlsson AS, LaBrie ST, Kinney AJ, von Wettstein-Knowles P, Browse J. A KAS2 cDNA complements the phenotypes of the Arabidopsis fab1 mutant that differs in a single residue bordering the substrate binding pocket. Plant J. 2002;29(6):761-70.

63. Shanklin J, Cahoon EB. Desaturation and related modifications of fatty Acids1. Annu Rev Plant Physiol Plant Mol Biol. 1998;49:611-41.

64. Kachroo A, Shanklin J, Whittle E, Lapchyk L, Hildebrand D, Kachroo P. The Arabidopsis stearoyl-acyl carrier protein-desaturase family and the contribution of leaf isoforms to oleic acid synthesis. Plant Mol Biol. 2007;63(2):257-71.

65. Schnurr JA, Shockey JM, de Boer GJ, Browse JA. Fatty acid export from the chloroplast: molecular characterization of a major plastidial acyl-coenzyme a synthetase from Arabidopsis. Plant Physiol. 2002;129(4):1700-9.

66. Zhao L, Katavic V, Li F, Haughn GW, Kunst L. Insertional mutant analysis reveals that long-chain acyl-CoA synthetase 1 (LACS1), but not LACS8, functionally overlaps with LACS9 in Arabidopsis seed oil biosynthesis. Plant J. 2010;64(6):1048-58.

67. Ruuska SA, Girke T, Benning C, Ohlrogge JB. Contrapuntal networks of gene expression during Arabidopsis seed fillingW. Plant Cell. 2002;14(6):1191-206.

68. Chen GQ, Turner C, He X, Nguyen T, McKeon TA, Laudencia-Chingcuanco D. Expression profiles of genes involved in fatty acid and triacylglycerol synthesis in castor bean (Ricinus communis L.). Lipids. 2007:42(3):263-74.

69. Lozinsky S, Yang H, Forseille L, Cook GR, Ramirez-Erosa I, Smith MA. Characterization of an oleate 12-desaturase from Physaria fendleri and identification of 5'UTR introns in divergent FAD2 family genes. Plant Physiol Biochem. 2014;75:114-22.

70. Cao S, Zhou XR, Wood CC, Green AG, Singh SP, Liu L, et al. A large and functionally diverse family of Fad2 genes in safflower (Carthamus tinctorius L). BMC Plant Biol. 2013;13:5.

71. Yang Q, Fan C, Guo Z, Qin J, Wu J, Li Q, et al. Identification of FAD2 and FAD3 genes in Brassica napus genome and development of allele-specific markers for high oleic and low linolenic acid contents. Theor Appl Genet. 2012;125(4):715-29.

72. Gidda SK, Shockey JM, Rothstein SJ, Dyer JM, Mullen RT. Arabidopsis thaliana GPAT8 and GPAT9 are localized to the ER and possess distinct ER retrieval signals: functional divergence of the dilysine ER retrieval motif in plant cells. Plant Physiol Biochem. 2009;47(10):867-79.

73. Kim HU, Li Y, Huang AH. Ubiquitous and endoplasmic reticulum-located lysophosphatidyl acyltransferase, LPAT2, is essential for female but not male gametophyte development in Arabidopsis. Plant Cell. 2005;17(4):1073-89.

74. Winter D, Vinegar B, Nahal H, Ammar R, Wilson GV, Provart NJ. An "Electronic Fluorescent Pictograph" browser for exploring and analyzing large-scale biological data sets. PLoS One. 2007;2(8):e718.

75. Arabidopsis eFP Brower. http://www.bar.utoronto.ca/.

76. Liu Q, Siloto RM, Lehner R, Stone SJ, Weselake RJ. Acyl-CoA:diacylglycerol acyltransferase: molecular biology, biochemistry and biotechnology. Prog Lipid Res. 2012;51(4):350-77.

77. Hernandez ML, Whitehead L, He Z, Gazda V, Gilday A, Kozhevnikova E, et al. A cytosolic acyltransferase contributes to triacylglycerol synthesis in sucrose-rescued Arabidopsis seed oil catabolism mutants. Plant Physiol. 2012;160(1):215-25.

78. Lozinsky S, Dauk M, Puttick D, Smith MA. Oilseed genomic resources: a Lesquerella Fendleri Est collection [abstract]. http://www.aaic.org/09progrm. htm 2009

79. Katavic V, Reed DW, Taylor DC, Giblin EM, Barton DL, Zou J, et al. Alteration of seed fatty acid composition by an ethyl methanesulfonate-induced mutation in Arabidopsis thaliana affecting diacylglycerol acyltransferase activity. Plant Physiol. 1995;108(1):399-409.

80. Hobbs DH, Lu C, Hills MJ. Cloning of a CDNA encoding diacylglycerol acyltransferase from Arabidopsis thaliana and its functional expression. FEBS Lett. 1999:452(3):145-9.

81. Routaboul JM, Benning C, Bechtold N, Caboche M, Lepiniec L. The TAG1 locus of Arabidopsis encodes for a diacylglycerol acyltransferase. Plant Physiol Biochem. 1999;37(11):831-40.

82. Jako C, Kumar A, Wei Y, Zou J, Barton DL, Giblin EM, et al. Seed-specific over-expression of an Arabidopsis cDNA encoding a diacylglycerol acyltransferase enhances seed oil content and seed weight. Plant Physiol. 2001;126(2):861-74.

83. Saha S, Enugutti B, Rajakumari S, Rajasekharan R. Cytosolic triacylglycerol biosynthetic pathway in oilseeds. Molecular cloning and expression of peanut cytosolic diacylglycerol acyltransferase. Plant Physiol. 2006;141(4):1533-43.

84. Cao H, Shockey JM, Klasson KT, Chapital DC, Mason CB, Scheffler BE. Developmental regulation of diacylglycerol acyltransferase family gene expression in tung tree tissues. PLoS One. 2013;8(10):e76946.

85. Xu J, Carlsson AS, Francis T, Zhang M, Hoffman T, Giblin ME, et al. Triacylglycerol synthesis by PDAT1 in the absence of DGAT1 activity is dependent on re-acylation of LPC by LPCAT2. BMC Plant Biol. 2012;12:4.

86. Bates PD, Fatihi A, Snapp AR, Carlsson AS, Browse J, Lu C. Acyl editing and headgroup exchange are the major mechanisms that direct polyunsaturated fatty acid flux into triacylglycerols. Plant Physiol. 2012;160(3):1530-9.

87. Wang L, Shen W, Kazachkov M, Chen G, Chen Q, Carlsson AS, et al. Metabolic interactions between the Lands cycle and the Kennedy pathway of glycerolipid synthesis in Arabidopsis developing seeds. Plant Cell. 2012;24(11):4652-69.

88. Lager I, Yilmaz JL, Zhou XR, Jasieniecka K, Kazachkov M, Wang P, et al. Plant acyl-CoA:lysophosphatidylcholine acyltransferases (LPCATs) have different specificities in their forward and reverse reactions. J Biol Chem. 2013;288(52):36902-14

89. Stahl U, Carlsson AS, Lenman M, Dahlqvist A, Huang B, Banas W, et al. Cloning and functional characterization of a phospholipid:diacylglycerol acyltransferase from Arabidopsis. Plant Physiol. 2004;135(3):1324-35.

90. Li R, Yu K, Hildebrand DF. DGAT1, DGAT2 and PDAT expression in seeds and other tissues of epoxy and hydroxy fatty acid accumulating plants. Lipids. 2010;45(2):145-57.

91. Zhang M, Fan J, Taylor DC, Ohlrogge JB. DGAT1 and PDAT1 acyltransferases have overlapping functions in Arabidopsis triacylglycerol biosynthesis and are essential for normal pollen and seed development. Plant Cell. 2009;21(12):3885-901.

92. Lin JT, Turner C, Liao LP, McKeon TA. Identification and quantification of the molecular species of acylglycerols in castor oil by HPLC using ELSD. J Liq Chromatogr Relat Technol. 2003;26(5):773-80.

93. Snapp AR, Kang J, Qi X, Lu C. A fatty acid condensing enzyme from Physaria fendleri increases hydroxy fatty acid accumulation in transgenic oilseeds of Camelina sativa. Planta. 2014;240(3):599-610.

94. Murashige T, Skoog F. A revised medium for rapid growth and bioassays with tobacco tissue cultures. Physiol Plant. 1962;15:473-97.

95. Pfaffl MW. A new mathematical model for relative quantification in real-time RT-PCR. Nucleic Acids Res. 2001;29(9):e45. 